\title{
Advances in immune therapies for the treatment of microsatellite instability-high/deficient mismatch repair metastatic colorectal cancer (Review)
}

\author{
KHALIL CHOUCAIR ${ }^{1}$, MALUKI RADFORD ${ }^{2}$, AJAY BANSAL $^{3}$, ROBIN PARK $^{4}$ and ANWAAR SAEED ${ }^{5}$ \\ ${ }^{1}$ Department of Medicine, Kansas University School of Medicine, Wichita, KS 67214; ${ }^{2}$ Department of Medicine, \\ Kansas University Medical Center; ${ }^{3}$ Department of Medicine, Division of Gastroenterology and Hepatology, \\ University of Kansas Medical Center and Kansas Cancer Institute, Kansas City, KS 66205; ${ }^{4}$ Department of Medicine, \\ MetroWest Medical Center/Tufts University School of Medicine, Framingham, Massachusetts, MA 01702; \\ ${ }^{5}$ Department of Medicine, Division of Medical Oncology, Kansas University Cancer Center, Kansas City, KS 66205, USA
}

Received May 14, 2021; Accepted July 23, 2021

DOI: 10.3892/ijo.2021.5254

\begin{abstract}
Microsatellite instability-high/deficient mismatch repair colorectal cancer (MSI-H/dMMR CRC) is a molecular subtype characterized by high-frequency mutations within DNA mismatch repair genes. Defects in the DNA mismatch repair machinery lead to subsequent frame-shift mutations, resulting in the generation of frame-shift peptides that serve as neoantigens. This has translated into exquisite sensitivity to immune checkpoint inhibitors (ICIs) and a significant clinical benefit from immune therapies in this patient population. The present article provides a comprehensive review of the advances in the field of immune therapies for MSI-H/dMMR metastatic CRC, with a focus on the major randomized clinical trials that led to Food and Drug Administration approval of specific ICIs for this population, a detailed review of the molecular background responsible for tumor response, as well as the mechanisms of resistance to ICI therapy. Finally, ongoing investigations of other immunotherapeutic strategies to address and overcome the challenges that currently limit response and long-term response to ICIs were presented.
\end{abstract}

\section{Contents}

1. Introduction

2. Immunotherapy in CRC: Molecular overview and rationale

3. Immunotherapy for MSI-H/dMMR mCRC: Review of clinical evidence and current recommendations

4. Mechanisms of resistance to ICIs in mCRC

5. Conclusions and future direction

Correspondence to: Dr Anwaar Saeed, Department of Medicine, Division of Medical Oncology, Kansas University Cancer Center, 2330 Shawnee Mission Pkwy, Kansas City, KS 66205, USA

E-mail: asaeed@kumc.edu

Key words: colorectal neoplasm, microsatellite instability, DNA mismatch repair, immunotherapy, immune checkpoint inhibitors

\section{Introduction}

According to the most recent global cancer statistics, colorectal cancer (CRC) is the second most common cause of cancer-associated death in males and females combined (1,2). In 2020, $\sim 1,1$ million new CRC cases were reported along with 576,858 deaths, accounting for nearly $10.0 \%$ of new cases and $5.8 \%$ of all cancer-associated deaths globally (1). While up to $10-15 \%$ of CRC patients carry one or more inherited pathogenic mutations associated with inherited syndromes (such as familial adenomatous polyposis or hereditary non-polyposis CRC) (3), $85 \%$ of $\mathrm{CRC}$ cases are sporadic and attributable to risk factors including age, race and sex, as well as modifiable risk factors including diet, tobacco use, diabetes and obesity (4). In the US, it is estimated that $\sim 20 \%$ of newly diagnosed CRC patients have metastatic disease (mCRC) at the time of presentation (2), of which 3-5\% harbor high microsatellite instability (MSI-H) and deficiency in mismatch repair mechanism (dMMR) of their genome $(5,6)$.

MSI is the result of somatic or germline mutations in the DNA mismatch repair (MMR) genes MutL homolog 1, MutS homolog 2 (MSH2), MSH6, post-meiotic segregation homolog 2 and epithelial cell adhesion molecule. This defect leads to frame-shift mutations due to the accumulation of DNA replication errors in the microsatellites of DNA coding regions. As a result, tumors with MSI-H/dMMR molecular profile tend to accumulate multiple insertion/deletion mutations that translate into frame-shift peptides (FSPs) expressed on tumor cell surfaces as neoantigens and recognized by the immune system. This has made MSI-H/dMMR mCRC attractive targets for immunotherapies that enhance self-immunity against cancer via exploitation of these neoantigens (7). Prior to the development of immune checkpoint inhibitors (ICIs), the mainstay of first-line therapy for mCRC was combination chemotherapy plus an anti-vascular endothelial growth factor (VEGF) or anti-epidermal growth factor antibody, depending on tumor characteristics without accounting for MSI-H/dMMR molecular status (8); yet, most patients progressed within 1 year of treatment with these systemic regimens (9). The introduction of ICIs, on the other hand, has since revolutionized cancer therapy and 
demonstrated impressive activity in patients with $\mathrm{mCRC}$ as well as other types of solid tumor that are MSI-H/dMMR $(7,10,11)$. In the present review, the molecular rationale behind the use of immunotherapy in patients with MSI-H/dMMR mCRC is described, available clinical data supporting its use are discussed and possible approaches and future directions to overcome current therapeutic challenges immunotherapy is facing in this select patient population are highlighted.

\section{Immunotherapy in CRC: Molecular overview and rationale}

ICIs: Mechanism of action. ICIs rely on the ability of tumor cells to suppress the innate immune system by exploiting the interaction between major histocompatibility complex (MHC)-T-cell receptor (TCR) and key ligands on the surface of tumor cells, known as immune checkpoints. These up-regulated immune checkpoints include programmed death 1 (PD-1), PD-1 ligand (PD-L1) and cytotoxic T-lymphocytes-associated protein 4 (CTLA-4), all of which act by inducing anergy or 'dampening' of the immune system (12-14).

PD-1 is expressed on the surface of T-cells, B-cells, dendritic cells and natural killer (NK) cells and becomes overexpressed in inflammatory microenvironments such as the tumor microenvironment (TME) (12). When PD-1 binds to PD-L1 on tumor cells, an inhibitory signaling cascade is initiated and results in i) direct inhibition of tumor cell apoptosis, ii) conversion of effector $\mathrm{T}$ cells into regulatory $\mathrm{T}$-cells and iii) kinase-dependent down-regulation of cytokine production required to stimulate proliferation and function of effector $\mathrm{T}$ cells $(12,13)$. This is the main mechanism by which tumor cells escape immune surveillance (15). Similarly, CTLA-4 is another co-inhibitory molecule expressed on tumor cells that functions as an immune checkpoint by binding to B7-1 (CD80) and B7-2 (CD86) on antigen-presenting cells, resulting in down-regulation of tumor-reactive T-cell activation, expansion and anti-tumor effects (14).

ICIs specifically target these checkpoints by disrupting the interaction between tumor-expressed inhibitory signals and cells of the immune system. ICIs were initially indicated to improve survival of patients with metastatic melanoma and non-small cell lung cancer (NSCLC) leading to the approval of ipilimumab (anti-CTLA-4), pembrolizumab and nivolumab (anti-PD-1) (16-20). These initial results were further consolidated with long-term follow-up studies revealing prolonged survival (for $\geq 10$ years) after treatment with ipilimumab (21). These robust responses have been explained by the high mutation prevalence commonly observed in both melanoma and NSCLC, suggesting that tumor cells with a high tumor mutation burden (TMB) generate more new peptides expressed as neoantigens on their MHC surface molecules; these neoantigens are recognized as non-self and result in priming of T-cell activation and cytotoxic killing $(22,23)$. More recently, the US Food and Drug Administration (FDA) approved the use of pembrolizumab for all solid tumors with high TMB, further consolidating the concept of neoantigenicity promoting response to ICIs (24).

ICIs in MSI-H/dMMR CRC: Mechanisms of response. A schematic of the mechanisms discussed below is provided in Fig. 1. In CRC specifically, the heterogeneous TME, which consists of immune cells, blood vessels, cytokines and growth factors, is rich in T-cells. Tumors with a greater T-cell infiltration have been associated with improved outcomes possibly via better immunologic control of tumor growth; in CRC, increased tumor-infiltrating lymphocytes (TILs) has been indicated to correlate with improved prognosis in terms of disease-free interval (25-27). However, the mere recognition of peptide-MHC class I complexes by the TCR alone is insufficient to efficiently activate T-cells without overcoming the co-inhibitory receptors discussed earlier and blocking the immune escape phenomenon. In fact, the use of ICI in patients with non-MSI-H/dMMR mCRC (95\% of all mCRC cases), was indicated to offer little to no clinical benefit [reviewed in (28)]. By contrast, ICIs have demonstrated impressive potency in patients with mCRC as well as other types of solid tumor that are MSI-H/dMMR $(7,10,11)$.

The increased sensitivity of MSI-H/dMMR mCRC tumors and their susceptibility to ICIs has been attributed to multiple immunologic, molecular and genetic factors. First, MSI-H/dMMR tumors have an abundance of TILs, specifically cytotoxic T lymphocytes, when compared with MMR proficient (pMMR) tumors (29). This observation has been linked to MSI-H tumors having a more favorable prognosis and disease course (30). While the mechanism behind this effect has remained to be fully elucidated, it has been hypothesized that the abundance of TILs creates an inflammatory TME that paradoxically does not eradicate the cancer but rather, triggers the up-regulation of several immune checkpoint molecules, including PD-1, PDL-1 and CTLA-4, in what appears an adaptive immune phenomenon that launches the immune escape mechanism discussed earlier (31). Part of this checkpoint-mediated immune escape adopted by cancer cells involves the increased conversion from effector T-cells to regulatory T-cells that secrete immunosuppressive molecules such as TGF- $\beta$, IFN- $\gamma$ and IL-10, ultimately resulting in immune anergy (31). IFN- $\gamma$ specifically has been indicated to up-regulate the expression of PD-L1, which is significantly higher in MSI-H tumors in comparison to microsatellite-stable (MSS) tumors (31). ICIs, by virtue of their mechanism of action, exploit these elevated levels of checkpoint inhibitors on cancer cells to disrupt the interaction between cancer cells and immune cells, ultimately 'blocking' the immune escape and reinvigorating the host's immune system. It is specifically the higher levels of checkpoint molecule expression in MSI-H tumors, originally adaptive to escape the host's immune system, that render this molecular subtype more sensitive and responsive to ICIs. Yet, high levels of PD-L1 would not solely explain this robust response to ICIs, as multiple studies have indicated that levels of PD-L1 expression alone are insufficient to predict response to ICIs, pointing towards the requirement for other molecular and genomic biomarkers [reviewed in (32)]. The TMB, which quantifies the total number of mutations present in a tumor specimen, has emerged as a promising quantitative genomic biomarker for response to ICIs, independent of the PD-L1 expression status [reviewed in (33)]. This becomes of unique relevance with MSI-H/dMMR mCRC that harbor a high level of somatic frame-shift mutations as a result of their dMMR mechanism. These molecular defects that translate into short 

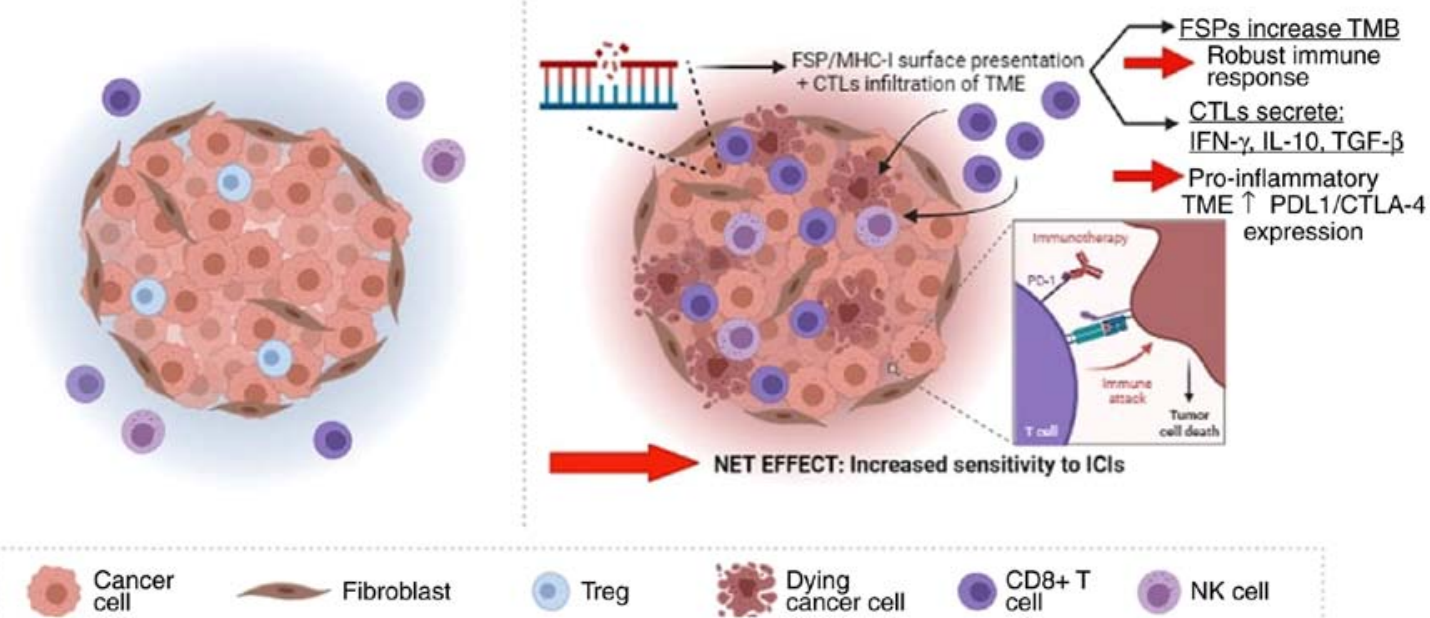

Treg
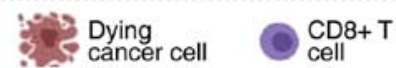

CD $8+T+1$
cell

NK cell

Figure 1. Mechanisms of sensitivity/response of MSI-H/dMMR CRC tumors to ICI therapy. dMMR results in the generation of FSPs that are presented as neoantigens via the MHC-1 system. Neoantigen recognition triggers the infiltration of CTLs and NK cells into the TME. CTLs secrete pro-inflammatory cytokines that promote the expression of PD-L1 and CTLA-4; these are subsequently recognized and inhibited by immune checkpoint inhibitors, leading to re-invigoration of T-cells that may attack and kill cancer cells. The presence of FSPs increases the TMB, resulting in a robust immune response to ICI. NK, natural killer; TMB, tumor mutational burden; TME, tumor microenvironment; FSPs, frame-shift peptides; CTLs, cytotoxic T lymphocytes; ICI, immune checkpoint inhibitor; MSI-H/dMMR CRC, microsatellite instability-high/deficient mismatch repair colorectal cancer; MSS, microsatellite-stable; pMMR, mismatch repair proficient; MHC, major histocompatibility complex; PD-L1, programmed death 1 ligand; CTLA-4, cytotoxic T-lymphocytes-associated protein 4; Treg, T-regulatory cell.

stretches of DNA (micro-satellites) serve as neoantigens (as FSPs), ultimately exposing cancer cells to the host's immune system, namely in the T-cell-infiltrated TME of MSI-H/dMMR tumors (7). The accumulation of neoantigens elicits a robust host immune response when recognized by the TILs (34), but also by involvement of macrophages and dendritic cells that serve as biologic immune intermediates for neoantigen presentation and delivery on the one hand, and as a pro-inflammatory vehicles that release inflammatory cytokines on the other hand, further enhancing the immunologic effect described earlier (35). In fact, MSI-H/dMMR tumors produce a significantly higher TMB in comparison to MSS tumors, in the realm of 10 -fold or higher. Numerous types of these mutations are sequences of tri-nucleotide repeats within the introns of protein-coding sequences (36). Of these mutations, the majority result in altered amino acid sequences and neoantigen peptides. Thus, patients with MSI-H/dMMR tumors represent a unique population of patients with mCRC that have been indicated to benefit the most from immune-based therapies (37). In a recent study by Valero et al (38), patients with MSI-H/dMMR with high TMB levels were reported to have better overall survival (OS) compared to MSS and this survival benefit was further prolonged in patients treated with ICIs compared to non-ICI therapy.

Finally, the impact of the MSI-H/dMMR status on triggering an immune response and predicting a durable response to ICIs reaches beyond the mere quantitative load of neoantigens to the actual identity and genomic function of the neo-peptides. An early study of the mutations causing amino acid alterations suggested that, within the selected CRC samples, there were roughly 7 unique epitopes that were involved in tumorigenesis (39). Early identified FSPs included transforming growth factor- $\beta$ receptor 2 (TGFBR2), phosphatase and tensin homolog, asteroid homolog 1, AIM2 and caspase 5 (36,39-42) and these FSPs, along with others such as HT001, AIM2 and TAF1B, were detected at significantly higher frequencies in MSI-H CRC compared to MSS (41).

More recent studies using serologic and bio-informatics approaches have further identified a wider range of FSPs and certain FSPs have potential functional genetic implications (43-46). In general, mutations in coding exons that are generated as a result of deficient MMR in MSI-H CRC result in complete functional inactivation (47). While functional validation is still pending for numerous FSPs, these observations open ways for novel mechanisms that explain the exquisite sensitivity of MSI-H/dMMR mCRC to ICIs: Beyond their immunogenicity as FSPs, certain mutated genes may further exert a functional anti-tumor effect that amplifies the immune efficiency of ICI in MSI-H/dMMR mCRC. For instance, loss of TGFBR 2 activity promotes an inflammatory response within the TME by inhibiting anti-inflammatory cytokines, increasing tumor-associated macrophage infiltration and NK T-cell activation (48). AIM2 on the other hand has been indicated to have tumor suppressive properties via promotion of an inflammatory response and inhibition of CRC cell proliferation and migration, similar to myristoylated alanine-rich protein kinase $\mathrm{C}$ substrate, another identified FSP that inhibits the proliferation of CRC (49-51). Inactivating mutation of these genes in MSI-H/dMMR $\mathrm{mCRC}$ may thus not result in promoting anti-tumor activity but may at least offer potential targets for precision therapy along with ICIs. The roles of other FSPs, such as TAF1B and ZNF294, remain to be identified $(47,52)$ while others such as HT001 are known to be non-coding with a strict immunogenic function (53). The extent to which such mutations and their resulting FSPs have genomic implications in ICI therapy warrants further study.

Yet, and irrespective of their potential with regard to genomic function, the identification of these FSPs that are 
present at higher frequencies in MSI-H/dMMR mCRC carries a significant potential for future vaccine development and other humoral FSP-based therapeutic strategies (42). With the mechanisms of response of MSI-H/dMMR to ICIs in mind, clinical evidence for the effectiveness of ICI for MSI has been provided in multiple studies, which is discussed in the following section.

\section{Immunotherapy for MSI-H/dMMR mCRC: Review of clinical evidence and current recommendations}

Evidence indicates that in patients with $\mathrm{mCRC}$, response to ICIs is limited to cases with MSI-H/dMMR molecular status $(54,55)$. In fact, the most recent practice guidelines across major National and International organizations recommend molecular testing for all newly diagnosed or recurrent cases of advanced and $\mathrm{mCRC}$ for MMR status and MSI markers (56-59). While those with pMMR are treated with systemic chemotherapy regimens, ICIs have been indicated for patients with MSI-H/dMMR who have failed systemic therapies and more recently, as an upfront therapy following an accelerated approval by the FDA as the 1st line therapy $(60,61)$. In this section, evidence from key phase II and III trials supporting the use of ICIs in MSI-H/dMMR mCRC, as well as ongoing trials, are reviewed (Table I).

Search methodology. A systematic review was conducted according to the PRISMA guidelines with the last update of the search performed on March 31, 2021. The search was conducted in PubMed as well as major conference proceedings (American Society of Clinical Oncology; European Society of Medical Oncology) using the following query terms: (colon cancer OR rectal cancer OR colorectal cancer OR colorectal neoplasm cancer) AND (MSI-H OR dMMR OR MSI-H/dMMR) OR (immunotherapy OR ICI OR immune therapy OR anti-PD-1 OR anti-PD-L1 OR anti-CTLA-4). In addition, the clinical trials registry (clinicaltrials.gov) was searched to identify ongoing trials that have with so far unpublished reports. Studies were included if they evaluated checkpoint inhibitors as a monotherapy or in combination with any other agent in a clinical trial setting in patients with MSI-H/dMMR mCRC. Studies were excluded if they evaluated checkpoint inhibitor therapy or systemic therapies for localized CRC or patients with MSS/pMMR, if a study was a protocol-only publication without data or if it reported overlapping data. In the latter case, the study with the most recent and/or most comprehensive data was included. The initial search identified a total of 29,980 studies. After review by title, abstract and full-text review, 9 studies were included in the final review (Table I). Furthermore, 28 additional ongoing and unpublished studies were identified via clinicaltrials. gov (Table II).

\section{Clinical evidence for anti-PDI therapy}

i) Pembrolizumab. Pembrolizumab is currently approved for chemo-refractory MSI-H/dMMR mCRC and as a 1st-line agent for this population (61). It was first evaluated in a phase II study (NCT01876511) involving patients with mCRC who had at least two or more previous chemotherapy regimens and consisting of three cohorts: $\mathrm{dMMR}(\mathrm{n}=11), \mathrm{pMMR}(\mathrm{n}=21)$ and nine patients with non-CRC dMMR gastrointestinal cancers (7). The primary endpoint was objective response rate (ORR) evaluated by 'Response evaluation criteria in solid tumors' (RECIST) v1.1. In the dMMR mCRC cohort, ORR was $40 \%$ compared to $0 \%$ in the pMMR cohort. No complete response (CR) was observed but patients with dMMR status had a high disease control rate (DCR) of 90\%, consisting of $40 \%$ partial responses (PR) and $50 \%$ of patients with stable disease (SD) when evaluated at 12 weeks. The median follow-up time was 36 weeks in the dMMR mCRC cohort and 20 weeks in the pMMR cohort, with a median progression-free survival (PFS) that was not reached in the dMMR group [vs. 2.2 months for the pMMR group; hazard ratio $(\mathrm{HR})=0.10$; $\mathrm{P}<0.001]$. At week 20, PFS rates were 78 and $11 \%$, respectively, and median OS was not reached in the dMMR group (vs. 5.0 months for pMMR mCRCs). In a follow-up study (KEYNOTE-016; NCT01876511) comprising 86 patients with different refractory dMMR cancers, 40 patients had dMMR mCRC (10). Compared to the previous study, the ORR was $52 \%$ but CR was achieved in $12 \%$ of patients with an average time to CR of 42 weeks. Neither median PFS nor OS were reached (median follow-up of 12.5 months) but later follow-up revealed a 2-year PFS of 59\% and 2-year OS of $72 \%(10,62)$. In a subsequent phase II study (KEYNOTE-164; NCT02460198) involving cases of MSI-H/dMMR unresectable advanced or mCRC, pembrolizumab was administered to patients who had received at least two prior therapies including fluoropyrimidine, oxaliplatin and irinotecan (cohort $A ; n=61)$ or at least one prior therapy (cohort $B ; n=63$ ) (62). The primary endpoint (ORR) was similar in both cohorts (33\%) but patients with lesser prior treatment (cohort B) had higher CR rates at $7.9 \%$ (vs. 3.3\%). It is worth noting that the ORR in this study (33\%) was smaller than that of KEYNOTE-016 (52\%) and this may be attributed to a smaller cohort size and the use of the immune-related RECIST (ir-RECIST) rather than RECIST in the latter study. Similarly, the median PFS was higher in cohort B (4.1 vs. 2.3 months) with an estimated 12-month PFS rate of $41 \%$ (vs. $34 \%$ in cohort A). As far as the median OS was concerned, it was not reached in the less pretreated cohort compared to 31.4 months in the group with more previous lines of treatment, with an estimated 1-year OS rate of 76 and $72 \%$, respectively. Besides confirming prior findings of a durable clinical benefit in pretreated MSI-H/dMMR mCRC, the study indicated a potential benefit from using anti-PD-1 in earlier stages of the disease. Based on pooled data from both the KEYNOTE-016 and -0164 trials ( $n=90$; pooled ORR: $36 \%$ ) the FDA approved pembrolizumab for patients with pretreated MSI-H/dMMR mCRC (63).

As a first-line treatment, pembrolizumab was evaluated in a phase III trial, in which investigators evaluated the efficacy and safety of pembrolizumab $(n=153)$ vs. investigator's choice of standard of care (SOC) chemotherapy $(n=154)$ in dMMR mCRC (KEYNOTE-177; NCT02563002) (64). The primary endpoint of the study was median PFS with significantly longer intervals in the pembrolizumab cohort (16.5 vs. 8.2 months; $\mathrm{HR}=0.6$ and $\mathrm{P}=0.0002$ ) at the median follow-up (28.4 months). This was also clinically meaningful with close to half of the patients $(48.3 \%)$ in the pembrolizumab arm without disease progression at 2 years, while patients experienced less drug-related adverse events compared to the SOC 


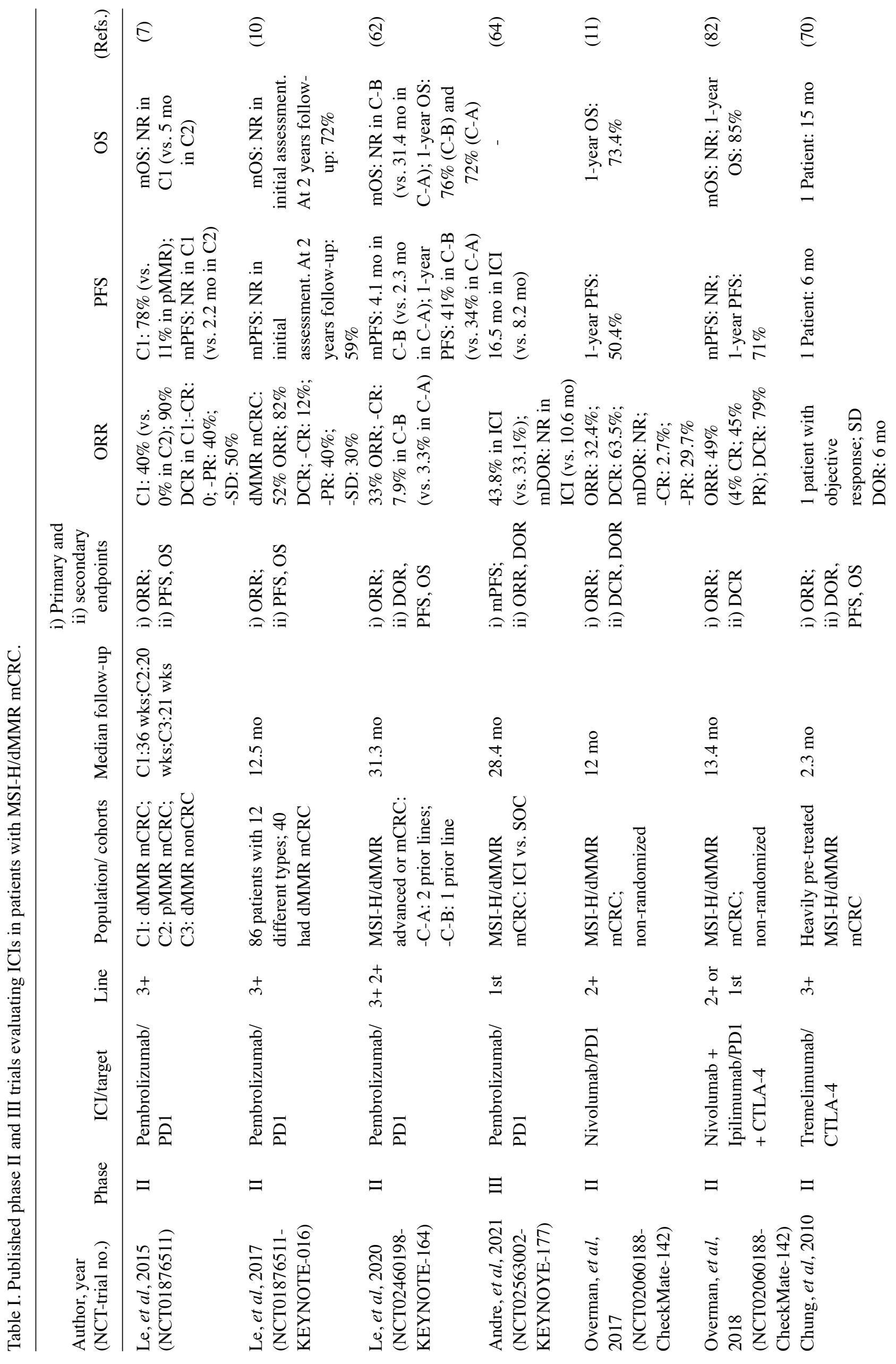




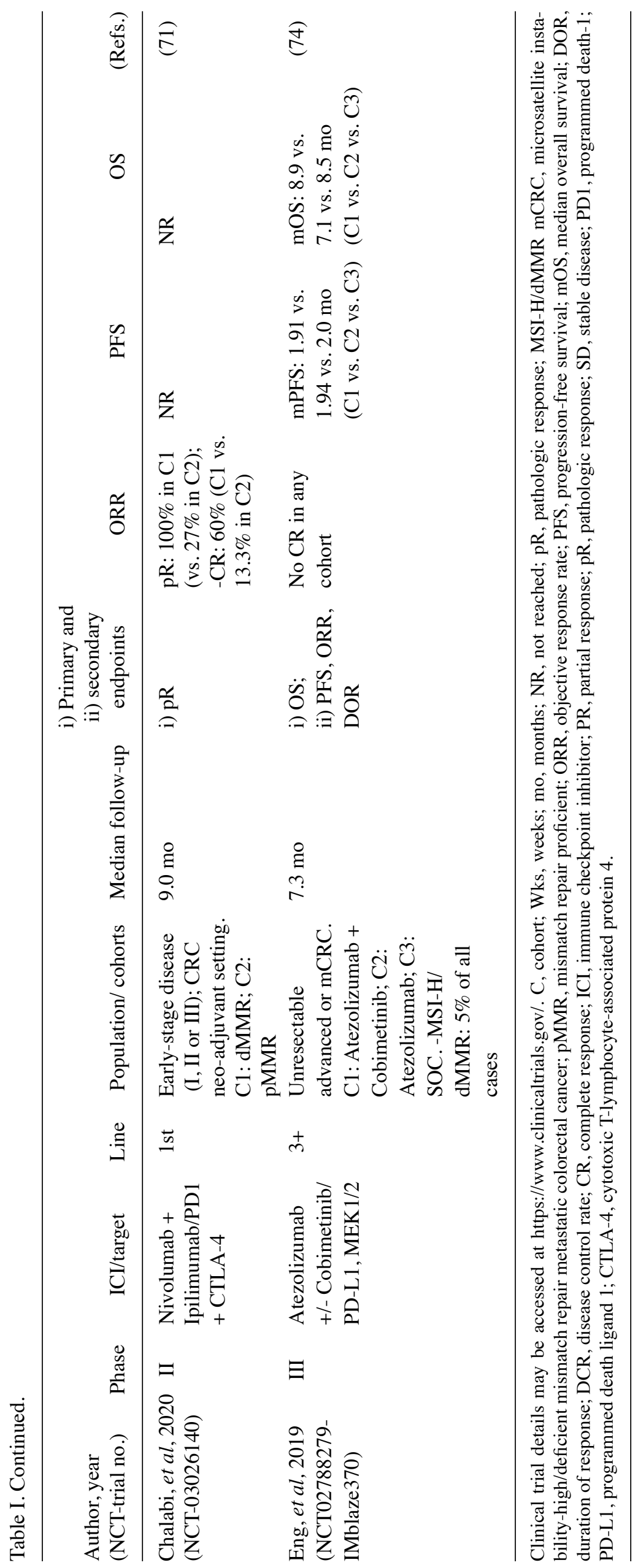




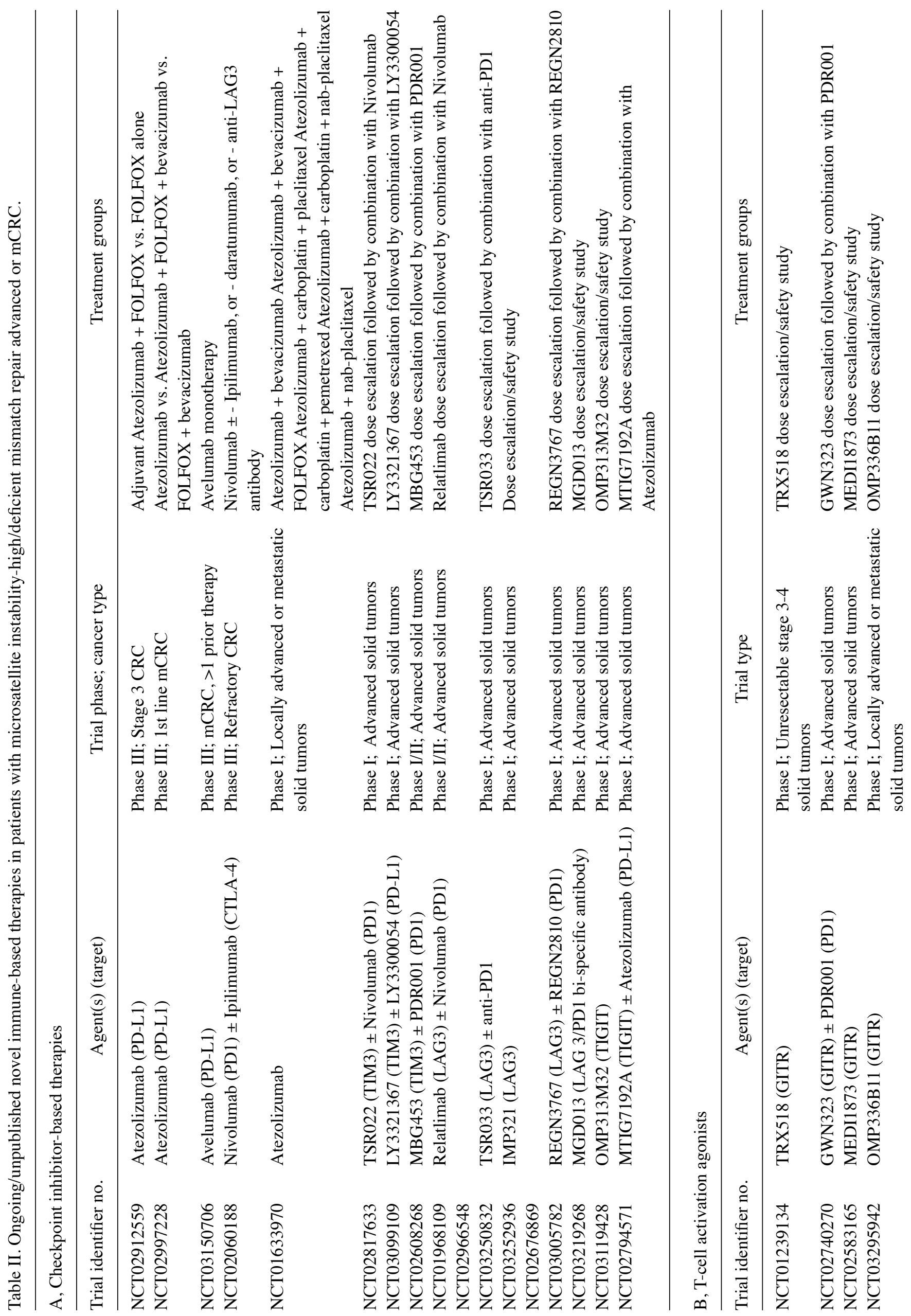




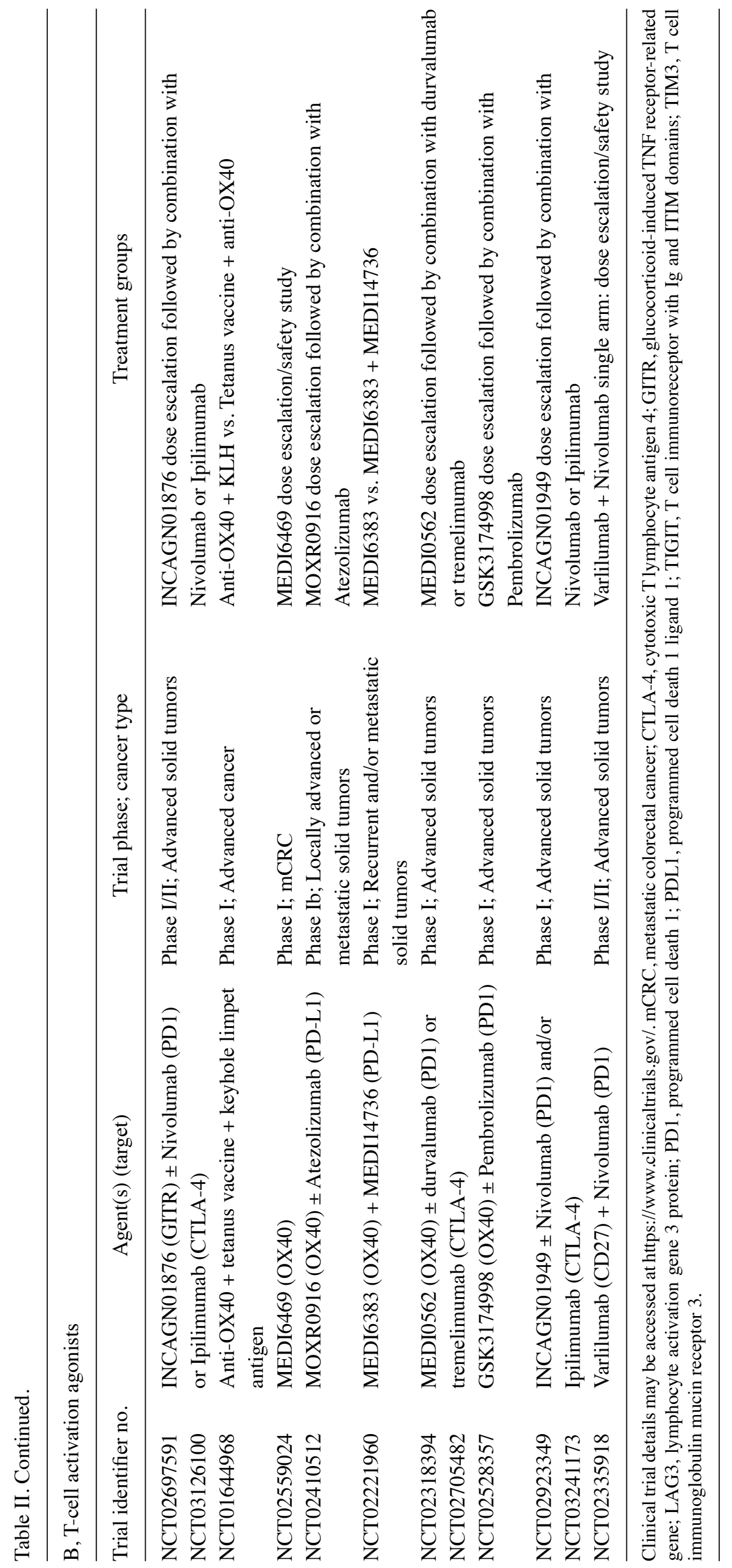


group (grade 2-5 toxicities; 22 vs. $66 \%$ respectively). Based on these results, the FDA approved pembrolizumab as a first-line treatment option for patients with MSI-H/dMMR mCRC (61). An updated analysis of the results with the final PFS, PFS-2 (time from randomization to progression on the next line of therapy or any cause of death), as well as a health-related quality of life (HR-QoL), was recently published (65). At the median follow-up of 32.4 months (range, 24.0-48.3 months), pembrolizumab continued to be superior to SOC for PFS with a median PFS of 16.5 months (vs. 8.2 months; HR 0.60; $\mathrm{P}=0.0002$ ), as well as 12- and 24-month PFS rates of 55.3 and $48.3 \%$ (vs. 37.3 and $18.6 \%$ with chemotherapy), respectively. The confirmed ORR was $43.8 \%$ (vs. $33.1 \%$ ) for pembrolizumab with longer durations of response (DOR; median DOR not reached vs. 10.6 months in SOC). Similarly, PFS-2 was longer with pembrolizumab (median not reached vs. 23.5 months; $\mathrm{HR}=0.63$ ) along with improved HR-QoL scores.

ii) Nivolumab. The clinical benefit of targeting PD-1 was also demonstrated with nivolumab. CheckMate-142 (NCT02060188) is a non-randomized phase II trial that evaluated nivolumab with or without ipilimumab (anti-CTLA-4) in MSI-H/dMMR mCRC patients that had been heavily pretreated (two or 3 previous lines of therapy) (11). In the initial part of the study, nivolumab was administered as monotherapy to 74 patients with ORR as a primary endpoint evaluated by RECIST v1.1. The results revealed an ORR of $32.4 \%$ with $2.7 \% \mathrm{CR}$ and $29.7 \%$ PR (central review). The median DOR was not reached, 1-year PFS was $50.4 \%$ and 1-year OS was 73.4\%. Disease control (defined as CR, PR or stable disease of 12 weeks or longer) was achieved by $63.5 \%$ of patients. This study was the first to demonstrate durable response and disease control with nivolumab in this patient population. Of note, the benefits of anti-PD-1 monotherapy were regardless of tumor PD-L1 expression, TILs status, mutational status (BRAF, KRAS) or germline dMMR status. Based on data from CheckMate-142, the FDA extended the approval of nivolumab for patients with MSI-H/dMMR mCRC who are refractory to 5-fluorouracil, oxaliplatin and irinotecan-based therapy (66).

In a second step of CheckMate-142, the efficacy of combining nivolumab with ipilimumab (anti-CTLA-4) was analyzed in 119 patients with dMMR mCRC who had received two or three lines of previous therapy (11). The rationale behind this combination is that both ICIs operate at different points of the immune response and when combined are able to act synergistically to promote the anti-tumor response (67). While the ORR as a primary endpoint reached $49 \%$ (4\% CR; $45 \%$ PR), the median PFS and OS were not reached, with one-year estimates of 71 and $85 \%$, respectively. The DCR for 12 weeks or longer and durable response for 12 months or longer was 79 and $83 \%$, respectively. The analysis of this cohort revealed better efficacy in combining nivolumab and ipilimumab compared to nivolumab monotherapy. Finally, the third cohort in CheckMate-142 consisted of untreated patients with MSI-H/dMMR who received nivolumab plus low-dose ipilimumab and the results revealed an ORR of $60 \%$, DCR of $84 \%$ and 12-month PFS of $77 \%$ (68). Those results further granted ipilimumab extended approval for use in patients with MSI-H/dMMR mCRC (69).
Clinical evidence for anti-CTLA-4 therapy. Unlike monotherapy with anti-PD1 agents, which has demonstrated clinical efficacy in the treatment of MSI-H/dMMR mCRC cases, treatment with single-agent anti-CTLA-4 monotherapy (ipilimumab or tremelimumab) failed to provide a clinical benefit in this population. In a single-arm phase II study of anti-CTLA-4 monotherapy with tremelimumab that involved 47 heavily pre-treated mCRC patients, 45 were considered response-evaluable, of which 44 did not reach the second dose of therapy (43 had progressive disease and 1 discontinued due to treatment-related adverse events) (70). Only one patient had stable disease for 6 months and the study did not demonstrate any clinically meaningful activity of single-agent tremilimumab. Thus, for now, anti-CTLA-4 therapy in MSI-H/dMMR $\mathrm{mCRC}$ is reserved for combined use with anti-PD-1 therapy, as discussed further above.

Of note, the nivolumab/ipilimumab combination has also been tested as a neo-adjuvant treatment for early CRC. In a recent phase Ib/II trial, nivolumab was administered in combination with ipilimumab to both dMMR $(n=20)$ and pMMR $(n=15)$ early-stage (I, II or III) CRC prior to surgery (71). In the dMMR cohort, $100 \%$ (20/20) achieved pathological response, with $60 \% \mathrm{CR}$ and $95 \%$ major responses (defined as $<10 \%$ residual tumor). Surprisingly, pMMR tumors also responded to the ICIs combination, albeit to a lesser extent (27\% pathological response, $13.3 \%$ CR, 6.7\% PR and $20 \%$ major responses). Regarding molecular markers, increased $\mathrm{CD} 8^{+} / \mathrm{PD}-1^{+} \mathrm{T}$-cell infiltration was predictive of response in these tumors.

Clinical evidence for anti-PD-L1 therapy. Atezolizumab targets the PD-L1 immune checkpoint. To date, there has not been any completed large clinical study in MSI-H/dMMR mCRC; however, early-phase studies involving anti-PD-L1 agents have been reported: In a phase I dose-escalation study of atezolizumab, one of the patients with unselected (unknown MSI or MMR status) mCRC achieved a durable PR (72). Another anti-PD-L1 monocloncal antibody (BMS-936559) was evaluated in 207 patients with advanced solid tumors, including 18 with unselected $\mathrm{mCRC}$ but no clinical response was observed (73).

Atezolizumab has also been evaluated in combination with SOC therapies. In a phase III trial (IMblaze370; NCT 02788279) performed by Eng et al (74), 363 patients with unresectable, locally advanced CRC or mCRC were treated with a combination of atezolizumab and cobimetinib/MEK $1 / 2$ inhibitor (cohort $1 ; n=183$ ) or atezolizumab monotherapy (cohort $2 ; \mathrm{n}=80$ ) or SOC regoraginib (cohort $3 ; \mathrm{n}=183$ ). Patients with dMMR tumors constituted $5 \%$ of all CRC cases. The results revealed no CR in any cohort along with low rates of PR with no difference between the 3 different cohorts. Similarly, no difference in PFS (1.91 vs. 1.94 vs. 2.0 months, respectively) or OS (8.9 vs. 7.1 vs. 8.5 months, respectively) was observed.

Atezolizumab has also been evaluated in a phase II study as a first-line treatment in unresectable wild-type BRAF mCRC in combination with fluoropyrimidine-bevacizumab treatment (MODUL; NCT02291289) (75). Compared to chemotherapy only, there was no improvement in PFS (HR=0.96) or OS ( $\mathrm{HR}=0.86$ ). 
Two large trials involving atezolizumab are currently underway: In the COMMIT trial (NCT02997228), a phase III study, patients newly diagnosed with $\mathrm{mCRC}(\mathrm{n}=373$, expected sample size) are randomly assigned to either receive FOLFOX with bevacizumab (control arm) with or without atezolizumab or atezolizumab monotherapy (76). The primary trial end-point was PFS, and secondary end-points included OS and ORR. This awaited study may potentially provide evidence for incorporating ICIs with SOC first-line agents in MSI-H/dMMR mCRC. Another ongoing phase III randomized trial, (ATOMIC ALLIANCE A021502; NCT02912559) is allocating stage III MSI-H/dMMR CRC to either SOC adjuvant chemotherapy monotherapy (FOLFOX for 6 months) or combined with atezolizumab, with an additional 6-months maintenance treatment with anti-PD-L1 (77). This study will investigate the ability of ICIs to eradicate any minimal residual disease in this patient population.

Patterns of response and safety profile of ICIs in MSI-H/dMMR $m C R C$. Beyond the marked efficacy in patients with MSI-H/dMMR mCRC, the use of ICIs in this patient population revealed certain unique patterns of response previously uncommonly observed with mCRC conventional therapy. Pseudo-progression is one unique striking pattern encountered in mCRC, also reported in melanoma, whereby an initial radiographic tumor enlargement is observed after ICI therapy is initiated, followed by measurable tumor regression months to years after therapy initiation $(78,79)$. This phenomenon may be explained by a transient increase in immune-cell tumor infiltration and pro-inflammatory cytokine release that generate edema (79). As a consequence, RECIST was modified to account for pseudo-progression through the development of immune-related response criteria (ir-RECIST) to assess responses to ICIs (80). In the trials reviewed above, RECIST was used for response assessment except in KEYNOTE-016, where ir-RECIST was used for primary endpoint assessment (10). This may explain why higher response rates were observed in KEYNOTE-016 compared to KEYNOTE-164 (where a pseudo-progression would be considered a progression as per the RECIST criteria).

Another aspect particular to ICIs in MSI-H/dMMR mCRC is the rate of CRs achieved compared to chemotherapy. In this population, $\mathrm{CR}$ rates with combination chemotherapy have been reported to be $1-2 \%(81)$, in contrast to $3 \%$ with pembrolizumab [median follow-up, 12.6 months; KEYNOTE-164; (62)], 3\% for nivolumab plus ipilimumab (median follow-up, 13.4 months; CheckMate-142) and 3\% for nivolumab monotherapy (median follow-up, 13 months) with an increase to $9 \%$ at a median follow-up of 21 months, thus achieving a deepening response over time [CheckMate-142; $(11,82)]$. These radiologic responses further translated to a clinical benefit as patients in CheckMate-142 who had CR or PR to nivolumab achieved a $100 \%$ 2-year OS compared to $50 \%$ among those with stable disease (83).

In addition to improved radiologic and clinical responses, the use of ICIs in mCRC has been unique in terms of DOR; for instance, the median DOR was not reached in CheckMate-142 after a median follow-up of 21 months (82). This has translated into prolonged median efficacy outcomes in MSI-H/dMMR mCRC in terms of median OS and 1-year OS as discussed earlier.
In terms of safety, ICIs have demonstrated a distinct safety profile compared to chemotherapy with toxicities relating to the skin (rash, pruritis), the gastrointestinal system (colitis) and endocrinopathies (thyroiditis, hypophysitis and adrenal insufficiency) (84). These toxicities are most likely secondary to autoimmune-like T-cell-mediated toxicities induced by disruption of the checkpoint's function. In the trials reviewed above, the most common immune-related toxicities (seen in at least $5 \%$ of patients receiving ICIs) included the following: Rash, pruritis, dry skin, diarrhea, colitis, nausea and vomiting, pancreatitis, gastritis, hepatic transaminitis, hypo/hyperthyroidism, hypophysitis, adrenal insufficiency, acute kidney injury, pneumonitis and myocarditis $(10,11,84)$. Most of these adverse events developed within 12 weeks of treatment initiation and resolved within 12 weeks of onset or were easily reversed by appropriate therapy. Compared to toxicities experienced with systemic therapy, ICIs offer not only a unique profile but also significantly better safety in terms of side effects.

Ongoing trials of novel immune modulators and immune-based combination therapies

Novel immune modulators. At present, PD1 and CTLA-4 blockade are the only FDA-approved ICIs for the treatment of MSI-H/dMMR mCRC. However, several other immune checkpoint interactions regulate T-cell activation in the TME. These include the following: T-cell immunoglobulin mucin receptor 3 (TIM3), lymphocyte activation gene 3 and T-cell Ig and ITIM domains; in pre-clinical studies, they have all been indicated to contribute to T-cell exhaustion and promotion of CRC progression $(85,86)$. These immune checkpoints are currently being evaluated in phase I clinical trials to assess their safety profile either as single agents or in combination with PD-L1 blockade (Table II) (87). Other agents with a different mechanism of action are also being evaluated; they act as antibody agonists of co-stimulatory immune receptors to enhance the host's immune system response against tumor cells. These include CD27, OX40 (CD134), 4-1BB (CD137) and glucocorticoid-induced TNF receptor-related gene (GITR; CD357 and CD40) (88).

Immune-based combination therapies. The use of ICIs in the treatment of MSI-H/dMMR mCRC has also been explored in combination with other conventional CRC therapies, including chemotherapy, targeted monoclonal antibodies (mAbs), radiation therapy (RT) and small-molecule tyrosine kinase inhibitors (TKIs). The rationale of combining ICIs with cytotoxic chemotherapy relies on evidence suggesting that cytotoxic cell killing results in cellular fragmentation that is taken up and presented to T-cells by antigen-presenting cells (89). Furthermore, chemotherapy-induced bone marrow suppression was indicated to decrease immune-suppressive T-regulatory cells as well as induce proliferation of other T-cells, resulting in stimulation of the immune system (90). Trials evaluating these treatments are summarized in Table II. Similar combinations are also being explored in patients with MSS/unknown MSS status mCRC [reviewed in (91)].

Another strategy to enhance the host immune response includes combining ICIs with mAbs that block growth 
factor receptors. In addition to this blockage, mAbs are thought to induce antibody-dependent cell-mediated cytotoxicity, hence justifying the above combination. However, current trials evaluating this combination are not specifically targeted to MSI-H/dMMR mCRC but rather serve as a potential strategy to sensitize the immune-insensitive MSS/pMMR CRC tumors. Small-molecule TKIs such as bevacizumab, an anti-VEGF molecule, have been evaluated in patients with MSI-H/dMMR mCRC. In preclinical studies, targeting VEGF has been demonstrated to offer potential therapeutic avenues for mCRC via suppression of tumor-associated macrophages, increase of interactions between dendritic cells and antigen-presenting cells, as well as augmenting endothelial vasculature to increase lymphocyte chemotaxis and T-cell tumor infiltration (92). This translated into a phase Ib clinical study that evaluated atezolizumab and bevacizumab combination in 10 pretreated patients with MSI-H/dMMR mCRC (93). At 11 months, the median OS had not been reached, the ORR was $30 \%$ and the median DOR was 7.8 months with 90\% DCR. The ongoing COMMIT trial discussed earlier (NCT02997228) is investigating the combination of bevacizumab with chemo-immunotherapy (mFOLFOX6 and atezolizumab) as a first-line therapy in patients with MSI-H/dMMR mCRC (76).

Finally, the combination of ICIs with RT has also been considered in the treatment of $\mathrm{mCRC}$ based on the abscopal effect theory, whereby radiation of cancer cells is thought to induce immunogenic cell death (with increased neo-antigen exposure), resulting in immune activation against tumor cells at more distant sites (94). This theory remains anecdotal with pre-clinical evidence [reviewed in (95)], as well as clinical evidence of ipilimumab/RT combination in melanoma achieving marked tumor regression at non-irradiated metastatic sites (96). This synergistic treatment modality has not been previously studied or explored in patients with MSI-H/dMMR mCRC but has been evaluated as a way of sensitizing MSS/pMMR mCRC to immunotherapy. In a phase II study (NCT02437071), pembrolizumab was evaluated in combination with RT demonstrating good tolerance, but modest effects in terms of response to ICI monotherapy with only one patient achieving PR (ORR, 4.5\%) (97). A subsequent study evaluated the effect of combining RT with dual ICIs: NSABP FC-9 (NCT02701400), a phase II single-arm study, evaluated the use of durvalumab (anti-PD-L1) plus tremelimumab (anti-CTLA-4) following hypofractionated $\mathrm{RT}$ in 20 patients with refractory MSS/pMMR mCRC (98). The initial results suggested a good overall safety profile and tolerance. Only two PRs were observed and lasted $>44$ weeks, along with 2 patients with stable disease for 12 and 16 weeks.

\section{Mechanisms of resistance to ICIs in mCRC}

Despite the marked responses to ICI compared to traditional therapies in patients with MSI-H/dMMR mCRC, up to $50 \%$ of patients ultimately acquire resistance to immunotherapy with subsequent progression and disease recurrence. While the MSI-H/dMMR status in itself, along with the TMB status, are powerful biomarkers of response to ICIs in mCRC, a requirement remains to understand the mechanisms by which tumor cells develop resistance to ICIs and eventually manage to re-escape the host's immune surveillance mechanism.

Resistance to ICIs has been described as either innate, termed as 'immunological ignorance' pertaining to natural lack of immune response to developing tumors, or acquired following treatment with ICIs (99). Innate resistance is frequently observed in patients with systemic immune-suppression (e.g. HIV patients) or in those that express few molecular targets that are recognized by the immune system (e.g. non-virally-induced tumors with low TMB expression, referred to as 'cold'). This is not the case in patients with MSI-H/dMMR mCRC with high TMB and a TME rich in immune infiltrate. Those patients respond well to ICIs but eventually develop acquired resistance to immunotherapy. Acquired resistance has been looked at as a by-product of two elements: i) The tumor's transcriptional profile or gene signature, but more extensively ii) the molecular type of the TME as classified by O'Donnell et al (99).

As far as the genomic signature is concerned, gene-set enrichment analysis studies revealed that transcriptional profiles enriched with IFN- $\gamma$ response genes are associated with better prognosis and response to anti-cancer immune therapies (100). Conversely, tumors with the innate anti-PD-1 resistance transcriptional profile lack any response to anti-PD-1 ICIs (101). In MSI-H/dMMR mCRC, IFN- $\gamma$ response genes are enriched (31), accounting for the significant response to ICIs. Other potential genomic markers of response/resistance among this patient population have been investigated and preliminary results did not indicate any predictive impact of the RAS/RAF mutational status, PD-L1 expression or the origin of the MMR deficiency status (inherited Lynch syndrome vs. acquired/sporadic origin) $(7,11,82,102)$. Numerous potential mechanisms of resistance are under investigation, including deleterious mutations in JAK, loss of MHC molecules or beta-2-microglobulin (B2M) loss-of-function mutations $(103,104)$. Similarly, the amount of extracellular mucin (assessed by histopathology) has been suggested to be associated with resistance to ICI (105).

In terms of types of TME, MSI-H/dMMR mCRC tumors belong to type $1 \mathrm{TME}$ which includes tumors with high TMB and an inflammatory gene signature. The three other types include different combinations of TMB levels along with presence/absence of inflammatory gene signature [reviewed in (99)]. The type of TME dictates the interaction between tumor cells and the immune system, and in type 1, high TMB levels and the presence of an inflammation gene signature are indicative of the existence of an ongoing but functionally suppressed immune response (106). Despite having the best probability of responding to the re-invigorative effect of ICIs, MSI-H/dMMR tumors exploit immune-suppressive/evasive strategies within the TME signaling pathway, which includes the following: Adaptive immune resistance pathways, loss of tumor antigen expression, insensitivity to interferons and cytokine/metabolite dysregulation (99). Understanding these mechanisms of resistance may provide additional potential targets to treat MSI-H/dMMR tumors. Table III summarizes the mechanisms of resistance to ICIs. 
Adaptive immune resistance. As mentioned in prior sections, elevated levels of type I and II interferons as well as immunosuppressive cytokines (IL-6, IL-12, TGF- $\beta$ ) lead to up-regulation of PD-L1 on immune cells and potentiate immune escape. While this interaction between PD-1 and PD-L1 is successfully targeted and disrupted by ICIs, the actual expression of PD-L1 is regulated in a complex manner beyond cytokine release and this includes influences by genomic alterations, transcriptional control mechanisms, mRNA stability as well as oncogenic signaling and protein stability (107). These alternative regulators of PD-L1 expression potentially represent novel targets to augment the efficacy of ICIs and extend the DOR. Similarly, it was indicated that tumors are able to release exosomes that express PD-L1, further contributing to immune suppression (108). Other adaptive immune resistance pathways have also been characterized, including overexpression of CD155 in both tumor cells and tumor-infiltrating myeloid cells (109). Binding of CD155 to CD96 and TGIT on tumor cells results in inhibition of the TILs and conversely, binding to CD266 on T-cells and NK cells competes with the prior inhibitory interaction (110). Thus, therapies targeting CD96 and TGIT may present a possible way to overcome resistance with evidence from preclinical tumor models demonstrating efficacy and synergy when PD-1 inhibition is combined with TGIT targeting (109-111).

Finally, the adaptive immune resistance to PD-1 inhibition has been indicated to be mediated through T-cell induced production of macrophage colony-stimulating factor 1 (MCSF-1), suggesting co-treatment of patients with anti-PD1 and MCSF-1 inhibitors as a possible strategy (112).

Loss of tumor antigen expression. Another mechanism for acquired resistance is the loss of the ability to present neoantigens, mostly via mutations affecting the antigen-presenting machinery such as proteasome subunits or transporters associated with antigen processing, or proteins involved in folding and sub-cellular translocation of MHC molecules (113). For instance, loss-of-function mutations of B2M, a chaperone protein essential for the folding and transport of MHC molecules to the cell surface, limits the recognition of tumor antigens by T-cells (114). This was clinically associated with resistance to ICIs $(101,104,115)$. Epigenetic events associated with tumor development and progression constitute another mechanism that alter human leukocyte antigen expression in tumor cells; unlike genetic alterations, epigenetic changes may potentially be reversed pharmacologically (113).

Insensitivity to IFNs. As indicated earlier, IFN- $\gamma$ is a major regulator of anti-tumor immunity that may also promote tumor resistance. Exposure of tumors to IFN- $\gamma$ in pre-clinical studies resulted in genetic instability in these tumors that translated into higher copy number variation associated with the DNA damage response and repair genes (116). However, IFN- $\gamma$ was also reported to induce neoantigen loss/down-regulation, thus favoring tumor escape $(116,117)$. Similarly, in tumor analysis from melanoma patients who responded poorly or acquired resistance to anti-CTLA-4 therapy, a higher frequency of mutations within genes involved in the IFN- $\gamma$ signaling pathway was noted and this included IFN- $\gamma$ receptor 1 (IFNGR1), IFNGR2, JAK2 and IFN regulatory factor $1(104,118)$. Similar observations of immune escape have also been made through loss of sensitivity to TNF (119). While the precise mechanisms behind this loss of sensitivity in both pathways have not been clearly defined, these observations suggest a potential benefit from genetic screening to identify new immunotherapy targets that operate in previously unknown pathways.

Cytokine and metabolite dysregulation. Hypoxia in TME promotes the accumulation of extra-cellular ATP that is metabolized to adenosine (120). One of the downstream effects of adenosine accumulation is the induction of strong immunosuppression within the TME: Adenosine signaling may impair effector T-cells and NK cells (120-122), and ADP-mediated immunosuppression through production of adenosine has been reported as a mechanism of tumor cell escape from PD-1/PD-L1 inhibition (123). Based on these observations, co-targeting enzymes involved in ATP degradation or blocking adenosine receptors may improve the efficacy of PD-1/PD-L1 inhibition (120). Tryptophan is another metabolite that mediates immunosupression when catabolised in the TME, via upregulation of indoleamine 2,3-dioxygenase 1 (IDO1), an IFN- $\gamma$-inducible enzyme (124). Preclinical work in cancer mouse models indicated that IDO promotes immunosuppression and IDO1 inhibitors had synergistic effects with ICIs (125). Despite promising early-phase trials of IDO1 inhibitors, the first phase III trial of an IDO1 inhibitor in combination with an anti-PD-1 antibody in melanoma patients did not achieve its primary endpoint (NCT02752074). However, the study still demonstrated the importance of concurrent biomarker and target development along with ICI development. In addition to upregulation of immunosuppressive metabolites, tumor cells frequently overexpress immunosuppressive cytokines, including VEGF and TGF- $\beta$. VEGF promotes upregulation of PD-1, CTLA-4 and TIM3 on TILs, thus promoting T-cell dysfunction $(126,127)$. Similarly, TGF- $\beta$ was indicated to upregulate PD-L1 expression and to promote metastasis of lung tumors, thus suggesting the potential of co-targeting PD-L1 and TGF- $\beta$ receptor (128).

\section{Conclusions and future direction}

Immunotherapy with checkpoint inhibitors has revolutionized the treatment of MSI-H/dMMR mCRC. This success is primarily based on the discovery of the immune escape phenomenon on the one hand and the advent of mAbs that block immune checkpoints and re-invigorate the immune system on the other hand. This strategy has proven successful in the MSI-H/dMMR population specifically based on this subtype's high mutation burden and its ability to present the FSP as neoantigens on MHC class I molecules to prime T-cells to recognize them as foreign. This has culminated in the FDA approval of anti-PD-L1 and anti-CTLA-4 ICIs for the treatment of MSI-H/dMMR mCRC. Despite this success, challenges have been encountered in primary non-responders and in those who develop acquired resistance to ICIs. As the current knowledge of the immune system and its intricacies continues to grow, 
Table III. Mechanisms of resistance to immune checkpoint inhibitor therapy in microsatellite instability-high/deficient mismatch repair colorectal cancer.

\begin{tabular}{ll} 
Mechanism of resistance & \multicolumn{1}{c}{ Description } \\
\hline Adaptive immune resistance & - Immunosuppressive cytokine-mediated upregulation of PD-L1 \\
& - Release of PD-L1 expressing exosomes \\
& - CD155 up-regulation on tumor cells inhibits TILs \\
& - MCSF-1 mediated immune resistance \\
& - Loss-of-function mutations in antigen-presenting machinery (e.g. B2M) \\
Loss of tumor antigen expression & - Epigenetic modulation of HLA expression in tumor cells \\
& - IFN- $\gamma$-mediated neo-antigen loss/down-regulation $\rightarrow$ immune escape \\
IFN- $\gamma$ insensitivity & - Mutations in IFN- $\gamma$ signaling pathway genes (IFNGR2, JAK2 and IRF1) $\rightarrow$ poor \\
& response to anti-CTLA-4 therapy in melanoma
\end{tabular}

Cytokine/metabolite mediated immune dysregulation

Adenosine-mediated

immunosuppression

Tryptophan mediated immune suppression

Tumor expressed immunosuppressive cytokines

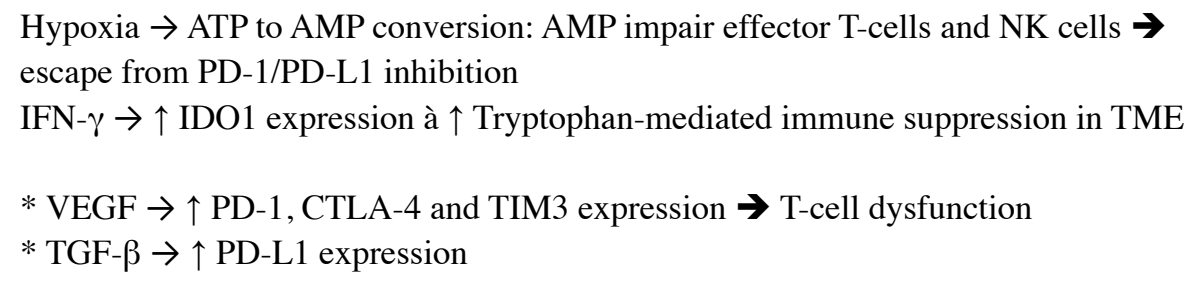

CTLA-4, cytotoxic T lymphocyte antigen 4; PD1, programmed cell death 1; PD-L1, programmed cell death 1 ligand 1; TIM3, T cell immunoglobulin mucin receptor 3; MCSF-1, macrophage colony-stimulating factor 1 ; HLA, human leukocyte antigen; IFN- $\gamma$, interferon- $\gamma$; ATP, adenosine triphosphate; AMP, adenosine monophosphate; NK cell, natural killer cell; IDO1, indoleamine 2,3-dioxygenase 1; TME, tumor microenvironment; VEGF, vascular endothelial growth factor; TGF- $\beta$, transforming growth factor- $\beta$; TILs, tumor-infiltrating lymphocytes; IFNGR2, IFN- $\gamma$ receptor 2; IRF1, IFN regulatory factor 1.

a better understanding of the different mechanisms behind immune resistance is gradually being achieved, allowing for the development of novel therapies, therapeutic combinations and strategies to overcome resistance.

At present, new-generation ICIs are being evaluated alone or in combination, with the hope of augmenting initial host anti-tumor immune response and enhance therapeutic efficacy. Other strategies include combination of ICIs with conventional therapy (chemotherapy, radiation, TKIs), as well as with new immune modulators. Furthermore, and based on the high frequency of generated FSPs in MSI-H/dMMR mCRC populations, efforts have been directed towards exploiting these mechanisms to develop individualized therapies, either by developing epitope-based vaccines or through harnessing adoptive cellular therapies via ex-vivo T-cell manipulation and chimeric antigen receptor T-cell generation. Although still in their early phases of clinical research, these novel approaches in conjunction with the ongoing trials outlined in the present article offer promising avenues to further advance immunotherapy in patients with MSI-H/dMMR mCRC.

\section{Acknowledgements}

Not applicable.

\section{Funding}

No funding was received.

\section{Availability of data and materials}

Data sharing is not applicable.

\section{Authors' contributions}

AS and AB conceived and designed the structure of this manuscript. KC and MR reviewed the literature and collated data from major studies and clinical trials. KC, MR and RP provided the initial draft of the manuscript, including the tables. AS, AB, KC and $\mathrm{RP}$ revised the manuscript for important intellectual content. All authors read and approved the final manuscript. Data authentication is not applicable.

\section{Ethics approval and consent to participate}

Not applicable.

\section{Patient consent for publication}

Not applicable.

\section{Competing interests}

AS reports research grants (to institution) for unrelated research work from Astrazeneca, Bristol Myers Squibb, Merck, Exelixis and Clovis and Advisory board/consultant fees from Astrazeneca, Bristol Myers Squibb, Merck and 
Pfizer. The remaining authors declare that they have no competing interests.

\section{References}

1. Sung H, Ferlay J, Siegel RL, Laversanne M, Soerjomataram I, Jemal A and Bray F: Global cancer statistics 2020: GLOBOCAN estimates of incidence and mortality worldwide for 36 cancers in 185 countries. CA Cancer J Clin 71: 209-249, 2021.

2. Siegel RL, Miller KD, Goding Sauer A, Fedewa SA, Butterly LF, Anderson JC, Cercek A, Smith RA and Jemal A: Colorectal cancer statistics, 2020. CA Cancer J Clin 70: 145-164, 2020.

3. Pearlman R, Frankel WL, Swanson B, Zhao W, Yilmaz A, Miller K, Bacher J, Bigley C, Nelsen L, Goodfellow PJ, et al: Prevalence and spectrum of germline cancer susceptibility gene mutations among patients with early-onset colorectal cancer. JAMA Oncol 3: 464-471, 2017.

4. Hull R, Francies FZ, Oyomno M and Dlamini Z: Colorectal cancer genetics, incidence and risk factors: In search for targeted therapies. Cancer Manag Res 12: 9869-9882, 2020.

5. Koopman M, Kortman GAM, Mekenkamp L, Ligtenberg MJ, Hoogerbrugge N, Antonini NF, Punt CJ and van Krieken JH: Deficient mismatch repair system in patients with sporadic advanced colorectal cancer. Br J Cancer 100: 266-273, 2009

6. Venderbosch S, Nagtegaal ID, Maughan TS, Smith CG, Cheadle JP, Fisher D, Kaplan R, Quirke P, Seymour MT, Richman SD, et al: Mismatch repair status and BRAF mutation status in metastatic colorectal cancer patients: A pooled analysis of the CAIRO, CAIRO2, COIN, and FOCUS studies. Clin Cancer Res 20: 5322-5330, 2014.

7. Le DT, Uram JN, Wang H, Bartlett BR, Kemberling H, Eyring AD, Skora AD, Luber BS, Azad NS, Laheru D, et al: PD-1 blockade in tumors with mismatch-repair deficiency. N Engl J Med 372: 2509-2520, 2015

8. Morse MA, Hochster $\mathrm{H}$ and Benson A: Perspectives on treatment of metastatic colorectal cancer with immune checkpoin inhibitor therapy. Oncologist 25: 33-45, 2020.

9. Chau I and Cunningham D: Treatment in advanced colorectal cancer: What, when and how? Br J Cancer 100: 1704-1719, 2009

10. Le DT, Durham JN, Smith KN, Wang H, Bartlett BR, Aulakh LK, Lu S, Kemberling H, Wilt C, Luber BS, et al: Mismatch repair deficiency predicts response of solid tumors to PD-1 blockade. Science 357: 409-413, 2017.

11. Overman MJ, McDermott R, Leach JL, Lonardi S, Lenz HJ Morse MA, Desai J,Hill A, Axelson M, Moss RA, et al: Nivolumab in patients with metastatic DNA mismatch repair-deficient or microsatellite instability-high colorectal cancer (CheckMate 142): An open-label, multicentre, phase 2 study. Lancet Oncol 18: 1182-1191, 2017.

12. Pardoll DM: The blockade of immune checkpoints in cancer immunotherapy. Nat Rev Cancer 12: 252-264, 2012.

13. Saunders PA, Hendrycks VR, Lidinsky WA and Woods ML: PD-L2:PD-1 involvement in $\mathrm{T}$ cell proliferation, cytokine production, and integrin-mediated adhesion. Eur J Immunol 35: 3561-3569, 2005.

14. Sharma P and Allison JP: Immune checkpoint targeting in cancer therapy: Toward combination strategies with curative potential Cell 161: 205-214, 2015.

15. Ijsselsteijn ME, Petitprez F, Lacroix L, Ruano D, van der Breggen R, Julie C, Morreau H, Sautès-Fridman C, Fridman WH and de Miranda NFDCC: Revisiting immune escape in colorectal cancer in the era of immunotherapy. Br J Cancer 120: 815-818, 2019.

16. Hodi FS, O'Day SJ, McDermott DF, Weber RW, Sosman JA, Haanen JB, Gonzalez R, Robert C, Schadendorf D, Hassel JC, et al: Improved survival with ipilimumab in patients with metastatic melanoma. N Engl J Med 363: 711-723, 2010.

17. Robert C, Long GV, Brady B, Dutriaux C, Maio M, Mortier L, Hassel JC, Rutkowski P, McNeil C, Kalinka-Warzocha E, et al: Nivolumab in previously untreated melanoma without BRAF mutation. N Engl J Med 372: 320-330, 2015.

18. Garon EB, Rizvi NA, Hui R, Leighl N, Balmanoukian AS, Eder JP, Patnaik A, Aggarwal C, Gubens M, Horn L, et al: Pembrolizumab for the treatment of non-small-cell lung cancer. N Engl J Med 372: 2018-2028, 2015.

19. Brahmer J, Reckamp KL, Baas P, Crinò L, Eberhardt WE, Poddubskaya E, Antonia S, Pluzanski A, Vokes EE, Holgado E, et al: Nivolumab versus docetaxel in advanced squamous-cell non-small-cell lung cancer. N Engl J Med 373: 123-135, 2015.
20. Borghaei H, Paz-Ares L, Horn L, Spigel DR, Steins M, Ready NE, Chow LQ, Vokes EE, Felip E, Holgado E, et al: Nivolumab versus docetaxel in advanced nonsquamous non-small-cell lung cancer. N Engl J Med 373: 1627-1639, 2015.

21. Schadendorf D, Hodi FS, Robert C, Weber JS, Margolin K, Hamid O, Patt D, Chen TT, Berman DM and Wolchok JD: Pooled analysis of long-term survival data from phase II and phase III trials of ipilimumab in unresectable or metastatic melanoma. J Clin Oncol 33: 1889-1894, 2015.

22. Rizvi NA, Hellmann MD, Snyder A, Kvistborg P, Makarov V, Havel JJ, Lee W, Yuan J, Wong P, Ho TS, et al: Mutational landscape determines sensitivity to PD-1 blockade in non-small cell lung cancer. Science 348: 124-128, 2015.

23. Schumacher TN and Schreiber RD: Neoantigens in cancer immunotherapy. Science 348: 69-74, 2015.

24. Subbiah V, Solit DB, Chan TA and Kurzrock R: The FDA approval of pembrolizumab for adult and pediatric patients with tumor mutational burden $(\mathrm{TMB}) \geq 10$ : A decision centered on empowering patients and their physicians. Ann Oncol 31: 1115-1118, 2020.

25. Mlecnik B, Tosolini M, Kirilovsky A, Berger A, Bindea G, Meatchi T, Bruneval P, Trajanoski Z, Fridman WH, Pagès F and Galon J: Histopathologic-based prognostic factors of colorectal cancers are associated with the state of the local immune reaction. J Clin Oncol 29: 610-618, 2011.

26. Galon J, Costes A, Sanchez-Cabo F, Kirilovsky A, Mlecnik B, Lagorce-Pagès C, Tosolini M, Camus M, Berger A, Wind P, et al: Type, density, and location of immune cells within human colorectal tumors predict clinical outcome. Science 313: 1960-1964, 2006.

27. Galon J, Fridman WH and Pagès F: The adaptive immunologic microenvironment in colorectal cancer: A novel perspective. Cancer Res 67: 1883-1886, 2007.

28. Franke AJ, Skelton WP, Starr JS, Parekh H, Lee JJ, Overman MJ, Allegra $\mathrm{C}$ and George TJ: Immunotherapy for colorectal cancer: A review of current and novel therapeutic approaches. J Natl Cancer Inst 111: 1131-1141, 2019.

29. Shia J, Holck S, DePetris G, Greenson JK and Klimstra DS: Lynch syndrome-associated neoplasms: A discussion on histopathology and immunohistochemistry. Fam Cancer 12: 241-260, 2013.

30. Choi YY, Bae JM, An JY, Kwon IG, Cho I, Shin HB, Eiji T, Aburahmah M, Kim HI, Cheong JH, et al: Is microsatellite instability a prognostic marker in gastric cancer? A systematic review with meta-analysis. J Surg Oncol 110: 129-135, 2014.

31. Llosa NJ, Cruise M, Tam A, Wicks EC, Hechenbleikner EM, Taube JM, Blosser RL, Fan H, Wang H, Luber BS, et al: The vigorous immune microenvironment of microsatellite instable colon cancer is balanced by multiple counter-inhibitory checkpoints. Cancer Discov 5: 43-51, 2015.

32. Lagos GG, Izar B and Rizvi NA: Beyond tumor PD-L1: Emerging genomic biomarkers for checkpoint inhibitor immunotherapy. Am Soc Clin Oncol Educ Book 40: 1-11, 2020.

33. Choucair K, Morand S, Stanbery L, Edelman G, Dworkin L and Nemunaitis J: TMB: A promising immune-response biomarker, and potential spearhead in advancing targeted therapy trials. Cancer Gene Ther 27: 841-853, 2020.

34. Bever KM and Le DT: An expanding role for immunotherapy in colorectal cancer. J Natl Compr Canc Netw 15: 401-410, 2017.

35. Vignali DA and Kuchroo VK: IL-12 family cytokines: Immunological playmakers. Nat Immunol 13: 722-728, 2012.

36. Kim TM, Laird PW and Park PJ: The landscape of microsatellite instability in colorectal and endometrial cancer genomes. Cell 155: 858-868, 2013.

37. Price TJ, Thavaneswaran S, Burge M, Segelov E, Haller DG, Punt CJ, Arnold D, Karapetis CS, Tebbutt NC, Pavlakis N, et al: Update on optimal treatment for metastatic colorectal cancer from the ACTG/AGITG expert meeting: ECCO 2015. Expert Rev Anticancer Ther 16: 557-571, 2016.

38. Valero C, Lee M, Hoen D, Zehir A, Berger MF, Seshan VE, Chan TA and Morris LGT: Response rates to anti-PD-1 immunotherapy in microsatellite-stable solid tumors with 10 or more mutations per megabase. JAMA Oncol 7: 739-743, 2021.

39. Segal NH, Parsons DW, Peggs KS, Velculescu V, Kinzler KW, Vogelstein B and Allison JP: Epitope landscape in breast and colorectal cancer. Cancer Res 68: 889-892, 2008.

40. Kloor M and von Knebel Doeberitz M: The immune biology of microsatellite-unstable cancer. Trends Cancer 2: 121-133, 2016.

41. Bauer K, Nelius N, Reuschenbach M, Koch M, Weitz J, Steinert G, Kopitz J, Beckhove P, Tariverdian M, von Knebel Doeberitz M and Kloor M: T cell responses against microsatellite instability-induced frameshift peptides and influence of regulatory T cells in colorectal cancer. Cancer Immunol Immunother 62: 27-37, 2013. 
42. Kloor M, Reuschenbach M, Pauligk C, Karbach J, Rafiyan MR, Al-Batran SE, Tariverdian M, Jäger E and von Knebel Doeberitz M: A frameshift peptide neoantigen-based vaccine for mismatch repair-deficient cancers: A phase I/IIa clinical trial. Clin Cancer Res 26: 4503-4510, 2020.

43. Reuschenbach $\mathrm{M}$, Kloor $\mathrm{M}$, Morak M, Wentzensen $\mathrm{N}$, Germann A, Garbe Y, Tariverdian M, Findeisen P, Neumaier M Holinski-Feder E and von Knebel Doeberitz M: Serum antibodies against frameshift peptides in microsatellite unstable colorectal cancer patients with Lynch syndrome. Fam Cancer 9: 173-179, 2010.

44. Ballhausen A, Przybilla MJ, Jendrusch M, Haupt S, Pfaffendorf E, Draxlbauer M, Seidler F, Krausert S, Ahadova A, Kalteis MS, et al The shared neoantigen landscape of MSI cancers reflects immunoediting during tumor evolution. bioRxiv: Jan 1, 2019 (Epub ahead of print)

45. Rus Bakarurraini NAA, Ab Mutalib NS, Jamal R and Abu N: The landscape of tumor-specific antigens in colorectal cancer. Vaccines (Basel) 8: 371, 2020.

46. Yang G, Zheng R and Jin Z: Correlations between microsatellite instability and the biological behaviour of tumours. J Cancer Res Clin Oncol 145: 2891-2899, 2019.

47. Kim NG, Rhee H, Li LS, Kim H, Lee JS, Kim JH, Kim NK and Kim H: Identification of MARCKS, FLJ11383 and TAF1B as putative novel target genes in colorectal carcinomas with microsatellite instability. Oncogene 21: 5081-5087, 2002.

48. Itatani Y, Kawada K and Sakai Y: Transforming growth factor- $\beta$ signaling pathway in colorectal cancer and its tumor microenvironment. Int J Mol Sci 20: 5822, 2019.

49. BickeböllerM,TagschererKE,Kloor M,JansenL,Chang-Claude J, Brenner H, Hoffmeister M, Toth C, Schirmacher P, Roth W and Bläker H: Functional characterization of the tumor-suppressor MARCKS in colorectal cancer and its association with survival. Oncogene 34: 1150-1159, 2015.

50. Hornung V, Ablasser A, Charrel-Dennis M, Bauernfeind F, Horvath G, Caffrey DR, Latz E and Fitzgerald KA: AIM2 recognizes cytosolic dsDNA and forms a caspase-1-activating inflammasome with ASC. Nature 458: 514-518, 2009.

51. Xu M, Wang J, Li H, Zhang Z and Cheng Z: AIM2 inhibits colorectal cancer cell proliferation and migration through suppression of Gli1. Aging (Albany NY) 13: 1017-1031, 2020.

52. Ivanov I, Lo KC, Hawthorn L, Cowell JK and Ionov $\mathrm{Y}$ : Identifying candidate colon cancer tumor suppressor genes using inhibition of nonsense-mediated mRNA decay in colon cancer cells. Oncogene 26: 2873-2884, 2007.

53. Tougeron D, Fauquembergue E, Rouquette A, Le Pessot F, Sesboüé R, Laurent M, Berthet P, Mauillon J, Di Fiore F Sabourin JC, et al: Tumor-infiltrating lymphocytes in colorectal cancers with microsatellite instability are correlated with the number and spectrum of frameshift mutations. Mod Pathol 22: $1186-1195,2009$.

54. Topalian SL, Hodi FS, Brahmer JR, Gettinger SN, Smith DC, McDermott DF, Powderly JD, Carvajal RD, Sosman JA, Atkins MB, et al: Safety, activity, and immune correlates of anti-PD-1 antibody in cancer. N Engl J Med 366: 2443-2454, 2012

55. Brahmer JR, Drake CG, Wollner I, Powderly JD, Picus J, Sharfman WH, Stankevich E, Pons A, Salay TM McMiller TL, et al: Phase I study of single-agent anti-programmed death-1 (MDX-1106) in refractory solid tumors: Safety, clinical activity, pharmacodynamics, and immunologic correlates. J Clin Oncol 28: 3167-3175, 2010.

56. Giardiello FM, Allen JI, Axilbund JE, Boland CR, Burke CA, BurtRW,Church JM,DominitzJA,JohnsonDA,Kaltenbach T, etal: Guidelines on genetic evaluation and management of Lynch syndrome: A consensus statement by the US multi-society task force on colorectal cancer. Gastroenterology 147: 502-526, 2014.

57. Stoffel EM, Mangu PB, Gruber SB, Hamilton SR, Kalady MF, Lau MW, Lu KH, Roach N and Limburg PJ; American Society of Clinical Oncology; European Society of Clinical Oncology: Hereditary colorectal cancer syndromes: American society of clinical oncology clinical practice guideline endorsement of the familial risk-colorectal cancer: European society for medical oncology clinical practice guidelines. J Clin Oncol 33: 209-217, 2015.

58. Luchini C, Bibeau F, Ligtenberg MJL, Singh N, Nottegar A, Bosse T, Miller R, Riaz N, Douillard JY, Andre F and Scarpa A: ESMO recommendations on microsatellite instability testing for immunotherapy in cancer, and its relationship with PD-1/PD-L1 expression and tumour mutational burden: A systematic review-based approach. Ann Oncol 30: 1232-1243, 2019.
59. Monahan KJ, Bradshaw N, Dolwani S, Desouza B, Dunlop MG, East JE, Ilyas M, Kaur A, Lalloo F, Latchford A, et al: Guidelines for the management of hereditary colorectal cancer from the British society of gastroenterology (BSG)/association of coloproctology of Great Britain and Ireland (ACPGBI)/United Kingdom cancer genetics group (UKCGG). Gut 69: 411-444, 2020.

60. Stein A, Moehler M, Trojan J, Goekkurt E and Vogel A: Immuno-oncology in GI tumours: Clinical evidence and emerging trials of PD-1/PD-L1 antagonists. Crit Rev Oncol Hematol 130: 13-26, 2018.

61. FDA approves pembrolizumab for first-line treatment of MSI-H/dMMR colorectal cancer. FDA, 2020.

62. Le DT, Kim TW, Van Cutsem E, Geva R, Jäger D, Hara H, Burge M, O'Neil B, Kavan P, Yoshino T, et al: Phase II open-label study of pembrolizumab in treatment-refractory, microsatellite instability-high/mismatch repair-deficient metastatic colorectal cancer: KEYNOTE-164. J Clin Oncol 38: 11-19, 2020.

63. US. Food and drug administration: KEYTRUDA (pembrolizumab) \{package insert\}, 2017

64. Andre T, Shiu KK, Kim TW, Jensen BV, Jensen LH, Punt CJA, Smith DM, Garcia-Carbonero R, Benavides M, Gibbs P, et al: Pembrolizumab versus chemotherapy for microsatellite instability-high/mismatch repair deficient metastatic colorectal cancer: The phase 3 KEYNOTE-177 study. J Clin Oncol 38 (18 Suppl): LBA4, 2020

65. Shiu KK, Andre T, Kim TW, Jensen BV, Jensen LH, Punt cJA, Smith DM, Garcia-Carbonero R, Benavides M, Gibbs P, et al: KEYNOTE-177: Phase III randomized study of pembrolizumab versus chemotherapy for microsatellite instability-high advanced colorectal cancer. J Clin Oncol 39 (3 Suppl): S6, 2021.

66. FDA grants nivolumab accelerated approval for MSI-H or dMMR colorectal cancer. FDA, 2017.

67. Das R, Verma R, Sznol M, Boddupalli CS, Gettinger SN, Kluger H, Callahan M, Wolchok JD, Halaban R, Dhodapkar MV and Dhodapkar KM: Combination therapy with anti-CTLA-4 and anti-PD-1 leads to distinct immunologic changes in vivo. J Immunol 194: 950-959, 2015.

68. Lenz HJJ, Van Cutsem E, Limon ML, Wong KY, Hendlisz A, Aglietta M, Garcia-Alfonso P, Neyns B, Luppi G, Cardin D, et al: Durable clinical benefit with nivolumab (NIVO) plus low-dose ipilimumab (IPI) as first-line therapy in microsatellite instability-high/mismatch repair deficient (MSI-H/dMMR) metastatic colorectal cancer (mCRC). Ann Oncol 29 (Suppl 8): viii714, 2018.

69. FDA grants accelerated approval to ipilimumab for MSI-H or dMMR metastatic colorectal cancer. FDA, 2018.

70. Chung KY, Gore I, Fong L, Venook A, Beck SB, Dorazio P, Criscitiello PJ, Healey DI, Huang B, Gomez-Navarro J and Saltz LB: Phase II study of the anti-cytotoxic T-lymphocyte-associated antigen 4 monoclonal antibody, tremelimumab, in patients with refractory metastatic colorectal cancer. J Clin Oncol 28: 3485-3490, 2010.

71. Chalabi M,Fanchi LF, Dijkstra KK, Van den Berg JG, Aalbers AG, Sikorska K, Lopez-Yurda M, Grootscholten C, Beets GL, Snaebjornsson P, et al: Neoadjuvant immunotherapy leads to pathological responses in MMR-proficient and MMR-deficient early-stage colon cancers. Nat Med 26: 566-576, 2020.

72. Herbst RS, Garon EB, Kim DW, Cho BC, Perez-Gracia JL, Han JY, Arvis CD, Majem M, Forster MD, Monnet I, et al: Long-term outcomes and retreatment among patients with previously treated, programmed death-ligand 1-positive, advanced non-small-cell lung cancer in the KEYNOTE-010 study. J Clin Oncol 38: 1580-1590, 2020 .

73. Brahmer JR, Tykodi SS, Chow LQ, Hwu WJ, Topalian SL, Hwu P, Drake CG, Camacho LH, Kauh J, Odunsi K, et al: Safety and activity of anti-PD-L1 antibody in patients with advanced cancer. N Engl J Med 366: 2455-2465, 2012.

74. Eng C, Kim TW, Bendell J, Argilés G, Tebbutt NC, Di Bartolomeo M, Falcone A, Fakih M, Kozloff M, Segal NH, et al: Atezolizumab with or without cobimetinib versus regorafenib in previously treated metastatic colorectal cancer (IMblaze370): A multicentre, open-label, phase 3, randomised, controlled trial. Lancet Oncol 20: 849-861, 2019.

75. Grothey A, Tabernero J, Arnold D, De Gramont A, Ducreux MP O'Dwyer PJ, Van Cutsem E, Bosanac I, Srock S, Mancao C, et al: Fluoropyrimidine (FP) + bevacizumab (BEV) + atezolizumab vs FP/BEV in BRAFwt metastatic colorectal cancer (mCRC): Findings from Cohort 2 of MODUL-a multicentre, randomized trial of biomarker-driven maintenance treatment following first-line induction therapy. Ann Oncol 29 (Suppl 8): viii714-viiii715, 2018. 
76. Lee JJ, Yothers G, Jacobs SA, Sanoff HK, Cohen DJ, Guthrie KA Henry NL, Ganz PA, Kopetz S, Lucas PC, et al: Colorectal cancer metastatic dMMR immuno-therapy (COMMIT) study (NRG-GI004/SWOG-S1610): A randomized phase III study of mFOLFOX6/bevacizumab combination chemotherapy with or without atezolizumab or atezolizumab monotherapy in the first-line treatment of patients with deficient DNA mismatch repair (dMMR) metastatic colorectal cancer. J Clin Oncol 36 (Suppl 15): TPS3615, 2018

77. Sinicrope FA, Ou FS, Shi Q, Nixon AB, Mody K, Levasseur A, Dueck AC, Dhanarajan AR, Lieu CH,Cohen DJ, et al: Randomized trial of FOLFOX alone or combined with atezolizumab as adjuvant therapy for patients with stage III colon cancer and deficient DNA mismatch repair or microsatellite instability (ATOMIC, Alliance A021502). J Clin Oncol 35 (Suppl 15): TPS3630, 2017.

78. Chiou VL and Burotto $M$ : Pseudoprogression and Immune-Related Response in Solid Tumors. J Clin Oncol 33: 3541-3543, 2015.

79. Solinas C, Porcu M, Hlavata Z, De Silva P, Puzzoni M, Willard-Gallo K, Scartozzi M and Saba L: Critical features and challenges associated with imaging in patients undergoing cancer immunotherapy. Crit Rev Oncol Hematol 120: 13-21, 2017.

80. Seymour L, Bogaerts J, Perrone A, Ford R, Schwartz LH, Mandrekar S, Lin NU, Litière S, Dancey J, Chen A, et al: iRECIST: Guidelines for response criteria for use in trials testing immunotherapeutics. Lancet Oncol 18: e143-e152, 2017.

81. Ducreux M, Bennouna J, Hebbar M, Ychou M, Lledo G, Conroy T, Adenis A, Faroux R, Rebischung C, Bergougnoux L, et al: Capecitabine plus oxaliplatin (XELOX) versus 5-fluorouracil/leucovorin plus oxaliplatin (FOLFOX-6) as first-line treatment for metastatic colorectal cancer. Int $\mathbf{J}$ Cancer 128 : 682-690, 2011

82. Overman MJ, Bergamo F, McDermott RS, Aglietta M, Chen F, Gelsomino F, Wong M, Morse M, Van Cutsem E, Hendlisz A, et al: Nivolumab in patients with DNA mismatch repair-deficient/microsatellite instability-high (dMMR/MSI-H) metastatic colorectal cancer (mCRC): Long-term survival according to prior line of treatment from CheckMate-142. J Clin Oncol 36 (Suppl 4): S554, 2018.

83. Overman MJ, Lonardi S, Wong KYM, Lenz HJ, Gelsomino F, Aglietta M, Morse MA, Van Cutsem E, McDermott R, Hill A, et al: Durable clinical benefit with nivolumab plus ipilimumab in DNA mismatch repair-deficient/microsatellite instability-high metastatic colorectal cancer. J Clin Oncol 36: 773-779, 2018

84. Brahmer JR, Lacchetti C, Schneider BJ, Atkins MB, Brassil KJ, Caterino JM, Chau I, Ernstoff MS, Gardner JM, Ginex P, et al: Management of immune-related adverse events in patients treated with immune checkpoint inhibitor therapy: American society of clinical oncology clinical practice guideline. J Clin Oncol 36: 1714-1768, 2018

85. Woo SR, Turnis ME, Goldberg MV, Bankoti J, Selby M, Nirschl CJ, Bettini ML, Gravano DM, Vogel P, Liu CL, et al Immune inhibitory molecules LAG-3 and PD-1 synergistically regulate T-cell function to promote tumoral immune escape. Cancer Res 72: 917-927, 2012.

86. Ngiow SF, von Scheidt B, Akiba H, Yagita H, Teng MW and Smyth MJ: Anti-TIM3 antibody promotes T cell IFN- $\gamma$-mediated antitumor immunity and suppresses established tumors. Cancer Res 71: 3540-3551, 2011.

87. Anderson AC, Joller N and Kuchroo VK: Lag-3, Tim-3, and TIGIT: Co-inhibitory receptors with specialized functions in immune regulation. Immunity 44: 989-1004, 2016.

88. Ward-Kavanagh LK, Lin WW, Šedý JR and Ware CF: The TNF receptor superfamily in co-stimulating and co-inhibitory responses. Immunity 44: 1005-1019, 2016.

89. Kroemer G, Galluzzi L, Kepp O and Zitvogel L: Immunogenic cell death in cancer therapy. Annu Rev Immunol 31: 51-72, 2013

90. Sun X, Suo J and Yan J: Immunotherapy in human colorectal cancer: Challenges and prospective. World J Gastroenterol 22: 6362-6372, 2016

91. Ganesh K, Stadler ZK, Cercek A, Mendelsohn RB, Shia J, Segal NH and Diaz LA Jr: Immunotherapy in colorectal cancer: Rationale, challenges and potential. Nat Rev Gastroenterol Hepatol 16: 361-375, 2019.

92. Limagne E, Euvrard R, Thibaudin M, Rébé C, Derangère V, Chevriaux A, Boidot R, Végran F, Bonnefoy $\mathrm{N}$, Vincent J, et al: Accumulation of MDSC and Th17 cells in patients with metastatic colorectal cancer predicts the efficacy of a FOLFOXbevacizumab drug treatment regimen. Cancer Res 76: 5241-5252, 2016.
93. Hochster HS, Bendell JC, Cleary JM, Foster P, Zhang W, He X, Hernandez G, Iizuka K, Eckhardt SG: Efficacy and safety of atezolizumab (atezo) and bevacizumab (bev) in a phase Ib study of microsatellite instability (MSI)-high metastatic colorectal cancer (mCRC). J Clin Oncol 35 (Suppl 4): S673, 2017.

94. Kaminski JM, Shinohara E, Summers JB, Niermann KJ, Morimoto A and Brousal J: The controversial abscopal effect. Cancer Treat Rev 31: 159-172, 2005.

95. Gaipl US, Multhoff G, Scheithauer H, Lauber K, Hehlgans S, Frey B and Rödel F: Kill and spread the word: Stimulation of antitumor immune responses in the context of radiotherapy. Immunotherapy 6: 597-610, 2014.

96. Postow MA, Callahan MK, Barker CA, Yamada Y, Yuan J, Kitano S, Mu Z, Rasalan T, Adamow M, Ritter E, et al: Immunologic correlates of the abscopal effect in a patient with melanoma. N Engl J Med 366: 925-931, 2012.

97. Segal NH, Kemeny NE, Cercek A, Reidy DL, Raasch PJ, Warren $\mathrm{P}$, Hrabovsky AE, Campbell N, Shia J, Goodman KA, et al: Non-randomized phase II study to assess the efficacy of pembrolizumab (Pem) plus radiotherapy (RT) or ablation in mismatch repair proficient (pMMR) metastatic colorectal cancer (mCRC) patients. J Clin Oncol 34 (Suppl 15): S3539, 2016.

98. Lee JJ, Yothers G, George TJ, Fakih MG, Mallick AB, Mitchell EP, Wade JL, Krauss JC, KayalehOR, Heron DE, et al: Abstract 2257: Phase II study of dual immune checkpoint blockade (ICB) with durvalumab (Durva) plus tremelimumab (T) following palliative hypofractionated radiotherapy (SBRT) in patients (pts) with microsatellite-stable (MSS) metastatic colorectal cancer (mCRC) progressing on chemotherapy: NSABP FC-9. Cancer Res 79 (Suppl 13): S2257, 2019.

99. O'Donnell JS, Long GV, Scolyer RA, Teng MWL and Smyth MJ: Resistance to PD1/PDL1 checkpoint inhibition. Cancer Treat Rev 52: 71-81, 2017.

100. Chen DS and Mellman I: Elements of cancer immunity and the cancer-immune set point. Nature 541: 321-330, 2017.

101. Hugo W, Zaretsky JM, Sun L, Song C, Moreno BH, Hu-Lieskovan S, Berent-Maoz B, Pang J, Chmielowski B, Cherry G, et al: Genomic and transcriptomic features of response to anti-PD-1 therapy in metastatic melanoma. Cell 165: 35-44, 2016

102. O'Neil BH, Wallmark JM, Lorente D, Elez E, Raimbourg J, Gomez-Roca C, Ejadi S, Piha-Paul SA, Stein MN, Abdul Razak AR, et al: Safety and antitumor activity of the anti-PD-1 antibody pembrolizumab in patients with advanced colorectal carcinoma. PLoS One 12: e0189848, 2017.

103. Kopetz S, Andre T, Overman MJ, Zagonel V, Lonardi S, Aglietta M, Gelsomino F, McDermott R, Wong KYM, Hendlisz A, et al: Abstract 2603: Exploratory analysis of Janus kinase 1 (JAK1) loss-of-function $(\mathrm{LoF})$ mutations in patients with DNA mismatch repair-deficient/microsatellite instability-high (dMMR/MSI-H) metastatic colorectal cancer (mCRC) treated with nivolumab + ipilimumab in CheckMate-142. Cancer Res 78 (Suppl 13): S2603, 2018.

104. Zaretsky JM, Garcia-Diaz A, Shin DS, Escuin-Ordinas H, Hugo W, Hu-Lieskovan S, Torrejon DY, Abril-Rodriguez G, Sandoval S, Barthly L, et al: Mutations associated with acquired resistance to PD-1 blockade in melanoma. N Engl J Med 375: 819-829, 2016.

105. Llosa NJ, Luber B, Siegel N, Awan AH, Oke T, Zhu Q, Bartlett BR, Aulakh LK, Thompson ED, Jaffee EM, et al: Immunopathologic stratification of colorectal cancer for checkpoint blockade immunotherapy. Cancer Immunol Res 7: 1574-1579, 2019.

106. Chen YP, Zhang Y, Lv JW, Li YQ, Wang YQ, He QM, Yang XJ, Sun Y, Mao YP, Yun JP, et al: Genomic analysis of tumor microenvironment immune types across 14 solid cancer types: Immunotherapeutic implications. Theranostics 7: 3585-3594, 2017.

107. Sun C, Mezzadra R and Schumacher TN: Regulation and function of the PD-L1 checkpoint. Immunity 48: 434-452, 2018.

108. Daassi D, Mahoney KM and Freeman GJ: The importance of exosomal PDL1 in tumour immune evasion. Nat Rev Immunol 20: 209-215, 2020.

109. Li XY, Das I, Lepletier A, Addala V, Bald T, Stannard K, Barkauskas D, Liu J, Aguilera AR, Takeda K, et al: CD155 loss enhances tumor suppression via combined host and tumor-intrinsic mechanisms. J Clin Invest 128: 2613-2625, 2018.

110. Blake SJ, Stannard K, Liu J, Allen S, Yong MC, Mittal D, Aguilera AR, Miles JJ, Lutzky VP, de Andrade LF, et al: Suppression of metastases using a new lymphocyte checkpoint target for cancer immunotherapy. Cancer Discov 6: 446-459, 2016. 
111. Harjunpää H, Blake SJ, Ahern E, Allen S, Liu J, Yan J, Lutzky V, Takeda K, Aguilera AR, Guillerey C, et al: Deficiency of host CD96 and PD-1 or TIGIT enhances tumor immunity without significantly compromising immune homeostasis. OncoImmunology 7: e1445949, 2018

112. Neubert NJ, Schmittnaegel M, Bordry N, Nassiri S, Wald N, Martignier C, Tillé L, Homicsko K, Damsky W, Maby-El Hajjami $\mathrm{H}$, et al: $\mathrm{T}$ cell-induced CSF1 promotes melanoma resistance to PD1 blockade. Sci Transl Med 10: eaan3311, 2018.

113. Seliger B: The link between MHC class I abnormalities of tumors, oncogenes, tumor suppressor genes, and transcription factors. J Immunotoxicol 11: 308-310, 2014.

114. Godfrey DI, Le Nours J, Andrews DM, Uldrich AP and Rossjohn J: Unconventional T cell targets for cancer immunotherapy. Immunity 48: 453-473, 2018

115. Sade-Feldman M, Jiao YJ, Chen JH, Rooney MS, Barzily-Rokni M, Eliane JP, Bjorgaard SL, Hammond MR, Vitzthum H, Blackmon SM, et al: Resistance to checkpoint blockade therapy through inactivation of antigen presentation. Nat Commun 8: 1136, 2017.

116. Takeda K, Nakayama M, Hayakawa Y, Kojima Y, Ikeda H, Imai N, Ogasawara K, Okumura K, Thomas DM and Smyth MJ: IFN- $\gamma$ is required for cytotoxic T cell-dependent cancer genome immunoediting. Nat Commun 8: 14607, 2017.

117. Dunn GP, Ikeda H, Bruce AT, Koebel C, Uppaluri R, Bui J, Chan R, Diamond M, White JM, Sheehan KC and Schreiber RD: Interferon-gamma and cancer immunoediting. Immunol Res 32 231-245, 2005.

118. Gao J, Shi LZ, Zhao H, Chen J, Xiong L, He Q, Chen T, Roszik J, Bernatchez C, Woodman SE, et al: Loss of IFN- $\gamma$ pathway genes in tumor cells as a mechanism of resistance to anti-CTLA-4 therapy. Cell 167: 397-404.e9, 2016.

119. Kearney CJ, Vervoort SJ, Hogg SJ, Ramsbottom KM Freeman AJ, Lalaoui N, Pijpers L, Michie J, Brown KK, Knight DA, et al: Tumor immune evasion arises through loss of TNF sensitivity. Sci Immunol 3: eaar3451, 2018.

120. Vijayan D, Young A, Teng MWL and Smyth MJ: Targeting immunosuppressive adenosine in cancer. Nat Rev Cancer 17: 709-724, 2017.
121. Ohta A, Gorelik E, Prasad SJ, Ronchese F, Lukashev D, Wong MK, Huang X, Caldwell S, Liu K, Smith P, et al: A2A adenosine receptor protects tumors from antitumor T cells. Proc Natl Acad Sci USA 103: 13132-13137, 2006.

122. Beavis PA, Divisekera U, Paget C, Chow MT, John LB, Devaud C, Dwyer K, Stagg J, Smyth MJ and Darcy PK: Blockade of A2A receptors potently suppresses the metastasis of $\mathrm{CD} 73^{+}$tumors. Proc Natl Acad Sci USA 110: 14711-14716, 2013.

123. Chen L, Diao L, Yang Y, Yi X, Rodriguez BL, Li Y, Villalobos PA, Cascone T, Liu X, Tan L, et al: CD38-mediated immunosuppression as a mechanism of tumor cell escape from PD-1/PD-L1 blockade. Cancer Discov 8: 1156-1175, 2018.

124. Triplett TA, Garrison KC, Marshall N, Donkor M, Blazeck J, Lamb C, Qerqez A, Dekker JD, Tanno Y, Lu WC, et al: Reversal of indoleamine 2,3-dioxygenase-mediated cancer immune suppression by systemic kynurenine depletion with a therapeutic enzyme. Nat Biotechnol 36: 758-764, 2018.

125. Moon YW, Hajjar J, Hwu P and Naing A: Targeting the indoleamine 2,3-dioxygenase pathway in cancer. J Immunother Cancer 3: 51, 2015.

126. Voron T, Colussi O, Marcheteau E, Pernot S, Nizard M, Pointet $A L$, Latreche $\mathrm{S}$, Bergaya $\mathrm{S}$, Benhamouda $\mathrm{N}$, Tanchot C, et al: VEGF-A modulates expression of inhibitory checkpoints on $\mathrm{CD}^{+} \mathrm{T}$ cells in tumors. J Exp Med 212: 139-148, 2015.

127. Johnston CJ, Smyth DJ, Dresser DW and Maizels RM: TGF- $\beta$ in tolerance, development and regulation of immunity. Cell Immunol 299: 14-22, 2016.

128. Strauss J, Heery CR, Schlom J, Madan RA, Cao L, Kang Z, Lamping E, Marté JL, Donahue RN, Grenga I, et al: Phase I trial of M7824 (MSB0011359C), a bifunctional fusion protein targeting PD-L1 and TGF $\beta$, in advanced solid tumors. Clin Cancer Res 24: 1287-1295, 2018.

(i) $\odot$ This work is licensed under a Creative Commons Attribution-NonCommercial-NoDerivatives 4.0 International (CC BY-NC-ND 4.0) License. 